\title{
Open partial isometries and positivity in operator spaces
}

\author{
by \\ David P. Blecher (Houston, TX) and Matthew Neal (Granville, OH)
}

\begin{abstract}
We first study positivity in $C^{*}$-modules using tripotents (= partial isometries) which are what we call open. This is then used to study ordered operator spaces via an "ordered noncommutative Shilov boundary" which we introduce. This boundary satisfies the usual universal diagram/property of the noncommutative Shilov boundary, but with all the arrows completely positive. Because of their independent interest, we also systematically study open tripotents and their properties.
\end{abstract}

1. Introduction. We are interested here in cones of positive operators $X_{+}=\{x \in X: x \geq 0\}$, for a space $X$ of bounded linear operators on a Hilbert space, where $\geq$ denotes the usual ordering of such operators. Besides the intrinsic interest of such objects (for example, operator positivity plays a central role in many areas of mathematical physics today), our work is a sequel to [13], which was a first step in a new approach to positivity in an operator space $X$, namely studying it in terms of the "noncommutative Shilov boundary" of $X$ (see $[6,26,12]$ ). The latter object is a Hilbert $C^{*}$-module, or, equivalently, a ternary ring of operators (or TRO for short), by which we will mean a closed subspace $Z$ of a $C^{*}$-algebra $A$ such that $Z Z^{*} Z \subset Z$. If $X$ contains positive operators, then so will any containing TRO. The starting point of the present investigation and [13] was the question of whether, in this case, all morphisms in the universal property of the noncommutative Shilov boundary can also be chosen to be positive (allowing this boundary to be used as a new tool in the study of ordered operator spaces). To answer this, one is led immediately to study positivity in TROs, and we

2000 Mathematics Subject Classification: Primary 46L08, 46A40, 47L07; Secondary 46B40, 46L07, 47B60, 47L05.

Key words and phrases: operator spaces, ordered spaces, noncommutative topology, open and closed projections, noncommutative Shilov boundary, TRO (ternary ring of operators), $J B^{*}$-triple.

Blecher was partially supported by grant DMS 0400731 from the National Science Foundation. Neal was supported by Denison University. 
address this topic first (the last section of our paper concerns positivity in general operator spaces). In [13] we considered the case of selfadjoint TROs $Z$ in a $C^{*}$-algebra $A$. In the first part of the present paper, we are able to generalize, to arbitrary TROs, a fundamental correspondence from [13]: we show that the natural cones in a TRO, namely $Z \cap A_{+}$in the notation above, are in a bijective correspondence with tripotents (= partial isometries) which are open $\left({ }^{1}\right)$ in the sense of [13]. The emphasis we place on the relation between positivity and the underlying algebra has its philosophical origin in [22]. Open tripotents generalize the notion of open projections in $C^{*}$-algebra theory [36], which in turn generalize the notion of open sets in topology. Since there appears to be no theory of general open tripotents (in our sense) in the literature, we give a careful development of this topic here. We also briefly discuss compact tripotents, a notion which has been treated in the literature in a more general setting (see e.g. [5, 15, 19, 21, 24]). We believe that these objects should play a role in operator space theory in the future, in view of the importance of TROs in that subject (see e.g. [12, Chapter 8] and references therein). For example, it has strong relations with the recent study of peak projections and peak tripotents $[27,10,9]$. In any case, our paper, like its predecessor, in some sense "marries" the notion of positivity of Hilbert space operators to ideas from the basic structure theory of $J B W^{*}$-triples.

Section 4 is mostly devoted to maximal orderings on TROs. For example, we analyze a conjectured characterization of maximal operator space orderings on $*$-TROs from [13]. Indeed, 1) we show that the proposed characterization is not true for all $*$-TROs, and 2) we isolate the precise class of $*$-TROs for which the characterization is true in general (we call these the completely orderable $*$-TROs).

In Section 5, we apply some of our theory from earlier sections to construct, for an ordered operator space $X$, an ordered version of the noncommutative Shilov boundary of $X$. More particularly, we assign to the usual noncommutative Shilov boundary of $X$ the natural cone associated with an open tripotent, which in turn is a supremum of certain "range tripotents" studied in Section 3. This "ordered boundary" answers the question raised at the start of this paper: it satisfies the usual universal diagram/property of the noncommutative Shilov boundary of $X$, but all the arrows are completely positive. We usually do not assume, unlike in the predecessor [13] and in the companion paper [11], that $X$ has an involution $*$. This is simply because of the greater generality and freedom available in our framework, and because the analogous results in the involutive case are in some sense just a special case (with some exceptions that are discussed in [11]). Our

$\left({ }^{1}\right)$ These are not the same as the open partial isometries of [19]. 
results yield, for example, a very algebraic characterization of the possible operator space orderings on a given operator space (see e.g. Theorem 5.5), and interesting facts about such orderings which are maximal. The results are particularly good for spaces $X$ whose positive cone densely spans $X$, which is a common assumption in the theory of ordered vector spaces. Indeed, it is often a very reasonable assumption since "order theory" can say very little about elements not in the span of the cone. In any case, it seems to be true that ordered operator spaces with densely spanning cones, which as far as we know have not hitherto been considered in the literature, constitute a setting to which much of the theory of (unital) operator systems generalizes in a natural and satisfactory way. We initiate the study of such spaces here and in the sequel [11], where, for example, we obtain a new unitization of such spaces (which is universal in that it has the biggest possible positive cone, as opposed to the unitization from [39, 40, 31] which has the smallest), and a striking rigidity property (see the end of Section 2 in [11]). This class certainly deserves further study in the future.

We now turn to precise definitions and notation. Any unexplained terms below can probably be found in [12], or any of the other recent books on operator spaces. All vector spaces are over the complex field $\mathbb{C}$. A given cone in a space $X$ will often be written as $X_{+}$, and we write $\geq$for the associated ordering: $x \geq y$ iff $x-y \in X_{+}$. Indeed, we will use the terms "cone" and "ordering" somewhat interchangeably. A matrix cone $\mathfrak{c}$ for us will simply be a sequence $\left(\mathfrak{c}_{n}\right)$, where $\mathfrak{c}_{n}$ is a cone in $M_{n}(X)$, such that if $\left[x_{i j}\right] \in \mathfrak{c}_{n}$ then $x_{i i} \in \mathfrak{c}_{1}$. A linear map $T: X \rightarrow Y$ between spaces with cones is positive if $T\left(X_{+}\right) \subset Y_{+}$. If the matrix spaces $M_{n}(X)$ and $M_{n}(Y)$ also each have a given cone, for each $n \in \mathbb{N}$, and if the canonical "amplification" $T_{n}$ : $M_{n}(X) \rightarrow M_{n}(Y)$ is positive for each $n \in \mathbb{N}$, then we say that $T$ is completely positive. A (resp. complete) order embedding is a (resp. completely) positive map $T$ such that $T^{-1}$ is (resp. completely) positive on $\operatorname{Ran}(T)$. An operator space ordering or operator space cone on an operator space $X$ is a specified matrix cone $\mathfrak{c}=\left(\mathfrak{c}_{n}\right)$ so that there exists a complete isometry $T$ from $X$ into a $C^{*}$-algebra $B$, which is at least completely positive. That is, $T$ is a complete isometry with $T_{n}\left(\mathfrak{c}_{n}\right) \subset M_{n}(B)_{+}$for all $n \in \mathbb{N}$. Of course, it is more natural in some sense to strengthen this last definition by also requiring $T$ to be a complete order embedding; and we remark that the ordered spaces satisfying this strengthened definition have been abstractly characterized in [39] as the "matrix ordered operator spaces" whose matrix norms coincide with the "modified numerical radius" norms (this follows from e.g. Corollary 4.11 there). Nonetheless, the convention we adopt seems to fit better with our results. Moreover, by the characterization from [39] just mentioned, one can easily see that the two definitions actually coincide for operator space cones (in our sense) also satisfying the mild conditions in [39, Definition 3.3]. 
We will sometimes be sloppy, and use $\mathfrak{c}$ interchangeably for $\mathfrak{c}_{1}$ and for the entire collection $\left\{\mathfrak{c}_{n}\right\}$, and vice versa. Similarly, $T(\mathfrak{c})$ often denotes $\left(T_{n}\left(\mathfrak{c}_{n}\right)\right)$. We say that one ordering on $X$ is majorized by another ordering if the positive cones for the first ordering are contained in the positive cones for the second ordering. We write $X^{\prime}$ for the dual Banach space (resp. dual operator space) of a Banach space (resp. operator space) $X$, and regard $X \subset X^{\prime \prime}$.

We refer to e.g. $[26,12]$ for the basic theory of TROs. TROs were characterized as operator spaces in [34], but we shall not need this here. A ternary morphism on a TRO $Z$ is a linear map $T$ such that $T\left(x y^{*} z\right)=$ $T(x) T(y)^{*} T(z)$ for all $x, y, z \in Z$. A tripotent is an element $u \in Z$ such that $u u^{*} u=u$. We order tripotents by $u \leq v$ if and only if $u v^{*} u=u$. This turns out to be equivalent to $u=v u^{*} u$, or to $u=u u^{*} v$, and implies that $u^{*} u \leq v^{*} v$ and $u u^{*} \leq v v^{*}[8]$. A WTRO is a weak* closed TRO in a $W^{*}$-algebra. We write $L(Z)$ for the linking $C^{*}$-algebra of a TRO; this has four "corners" $Z Z^{*}, Z, Z^{*}$, and $Z^{*} Z$. Here $Z Z^{*}$ is the closure of the linear span of products $z w^{*}$ with $z, w \in Z$, and similarly for $Z^{*} Z$. If $E$ is a WTRO, then we write $\bar{L}(E)$ for the $W^{*}$-algebra linking algebra; this has four "corners" $\overline{E E^{*}}{ }^{\text {weak }}{ }^{*}, E, E^{*}$, and ${\overline{E^{*}}}^{E^{\text {weak* }}}$. The second dual of a TRO $Z$ is a WTRO, and $L(Z)^{\prime \prime}=\bar{L}\left(Z^{\prime \prime}\right)$ (see e.g. the proof of $[12,8.5 .17]$ ). We will denote by $I$ the injection from $Z$ into $L(Z)$ given by $I(z)=z \otimes e_{12}+z^{*} \otimes e_{21}$. Note that $I(z)^{2}=z z^{*} \otimes e_{11}+z^{*} z \otimes e_{22}$. For a tripotent $u$, we set $\widehat{u}=\frac{1}{2}\left(I(u)+I(u)^{2}\right)$, and $\breve{u}=\frac{1}{2}\left(-I(u)+I(u)^{2}\right)$; these are projections. Define $\Theta: L(Z) \rightarrow L(Z)$ to be the period $2 *$-automorphism which changes the sign of the off-diagonal entries. Note that $\breve{u}=\Theta^{\prime \prime}(\widehat{u})$. We say that a projection $r \in L(Z)^{\prime \prime}$ is antisymmetric if $r \perp \Theta^{\prime \prime}(r)$, or equivalently, if $r=\widehat{v}$ for a tripotent $v \in Z^{\prime \prime}$ (see Lemma 3.6).

A subTRO of a TRO $Z$ is a closed subspace of $Z$ which is closed under the ternary product $x y^{*} z$. We write $\langle\mathcal{S}\rangle$ for the smallest subTRO containing a given subset $\mathcal{S}$ of $Z$. An inner ideal (resp. ternary ideal) of $Z$ is defined to be a closed subspace $J$ with $J Z^{*} J \subset J$ (resp. $J Z^{*} Z \subset J$ and $Z Z^{*} J \subset J$ ). Clearly inner and ternary ideals are subTROs. A $*$-TRO is a selfadjoint TRO $Z$ in a $C^{*}$-algebra $B$, and by an inner $*$-ideal or ternary $*$-ideal we mean an inner or ternary ideal which is selfadjoint (that is, closed under the involution). A tripotent in a $*$-TRO is selfadjoint if $u=u^{*}$, and central if $u z=z u$ for all $z \in Z$.

The Peirce 2-space of a tripotent $u$ in a TRO $Z$ is the subset

$$
Z_{2}(u)=\left\{z \in Z: z=u u^{*} z u^{*} u\right\}=u u^{*} Z u^{*} u=u Z^{*} u .
$$

Clearly $Z_{2}(u)$ is an inner ideal of $Z$, and if $Z$ is a WTRO then it is weak* closed. There is a natural product (namely $x \cdot y=x u^{*} y$ ) and involution 
(namely $x^{\sharp}=u x^{*} u$ ) on $Z_{2}(u)$ making the latter space into a unital $C^{*}$ algebra. The identity element of course is $u$. If $u \leq v$ then $Z_{2}(u)$ is a hereditary $C^{*}$-subalgebra of $Z_{2}(v)$, and $u$ becomes a projection in the last algebra. If $Z$ is a WTRO then $Z_{2}(u)$ is a $W^{*}$-algebra. The positive cone in the $C^{*}$ algebra $Z_{2}(u)$ will be written as $\mathfrak{c}_{u}$. Strictly speaking, we should probably write $\mathfrak{c}_{u}^{Z}$ for this cone, but to avoid excessive notation we will write the simpler expression. We leave it to the reader to make sense of the space which $\mathfrak{c}_{u}$ lives in (it will always be the TRO that $u$ belongs to). It is easy to check that $u^{*} Z_{2}(u)$ is a $C^{*}$-subalgebra of $Z^{*} Z$, and the map $z \mapsto u^{*} z$ is a $*$-isomorphism from $Z_{2}(u)$, with the product and involution above, onto this $C^{*}$-subalgebra. From this it is easy to see that

$$
\mathfrak{c}_{u}=\left\{z \in Z_{2}(u): u^{*} z \geq 0\right\}=\left\{z \in Z: u^{*} z \geq 0, z=u z^{*} u\right\},
$$

and also equals $\left\{u u^{*} z u^{*} u z^{*} u: z \in Z\right\}$, where these inequalities are in the $C^{*}$-algebra $Z^{*} Z$. If $u \in Z^{\prime \prime}$, we define $\mathfrak{d}_{u}$ to be the cone $\mathfrak{c}_{u} \cap Z$ in $Z$. We also write $\mathfrak{c}_{u}^{\prime}$ for the weak* closure of $\mathfrak{d}_{u}$ in $Z^{\prime \prime}$. In contrast to $\mathfrak{c}_{u}$, the cone $\mathfrak{d}_{u}$ lies in $Z$, and not in the space $Z^{\prime \prime}$ which $u$ lies in in this case. Finally, we will write $Z(u)$ for $Z_{2}^{\prime \prime}(u) \cap Z$. Following [13], we say that a tripotent $u$ in the WTRO $Z^{\prime \prime}$ is open if when we consider $Z_{2}^{\prime \prime}(u)$ as a $W^{*}$-algebra in this way, then $u$ is the weak* limit in $Z^{\prime \prime}$ of an increasing net from $\mathfrak{d}_{u}=\mathfrak{c}_{u} \cap Z$. Beware that this definition differs from the one given in [19] (for example, all unitaries are open in the sense of that paper). We will show that the spaces $\mathfrak{d}_{u}$, for open tripotents $u$, are exactly the natural cones in $Z$, and that this sets up an order preserving bijection between open tripotents and natural cones.

Lemma 1.1. A positive ternary morphism between $C^{*}$-algebras is a $*$-homomorphism, and hence it is completely positive. In particular, a positive linear completely isometric surjection between $C^{*}$-algebras is a $*$-isomorphism.

Proof. The first assertion may be found in the proof of [13, Corollary 4.3(2)]. The second we shall not need (it is stated as background), and it follows from the well known fact that the surjective linear complete isometries between TROs are exactly the ternary isomorphisms.

2. Open tripotents and natural cones. We begin with the following simple but fundamental observation, which we have not seen in the literature:

Lemma 2.1. Let $Z$ be a TRO inside a $C^{*}$-algebra $A$. Then the subspace $J(Z)=Z \cap Z^{*} \cap Z^{*} Z \cap Z Z^{*}$ is a $C^{*}$-subalgebra of $A$ which is also an inner ideal in $Z$. Moreover, the positive cone $J(Z)_{+}$of this $C^{*}$-subalgebra equals $Z \cap A_{+}$. 
Proof. The proof is left to the reader, except for the last assertion. Clearly $J(Z)_{+} \subset Z \cap A_{+}$. Conversely, if $x \in Z \cap A_{+}$then of course $x \in Z^{*}$. Also, $x^{2} \in Z^{*} Z$, so that $x \in Z^{*} Z$ since square roots remain in a $C^{*}$-algebra. Similarly, $x \in Z Z^{*}$, so that $x \in J(Z)$.

The positive cone $J(Z)_{+}$will be called a natural cone for $Z$, and the corresponding ordering on $Z$ is called a natural ordering. Since $M_{n}(Z)$ is a TRO in $M_{n}(A)$, of course one has a sequence of cones, $M_{n}(J(Z))_{+}=$ $J\left(M_{n}(Z)\right)_{+}$, but since the cone $J(Z)_{+}$determines the others (see Corollary 2.2 ), it will not often be necessary to mention these other cones. Thus we often suppress the obvious facts concerning them (in Section 4 we will start to be more careful in this regard). We also use the term "natural cone" even when the $C^{*}$-algebra $A$ is not in evidence. Thus, a cone $\mathfrak{d}$ in $Z$ is natural if there exists a one-to-one ternary morphism $\varphi: Z \rightarrow B$, for a $C^{*}$-algebra $B$, such that $\varphi(\mathfrak{d})=\varphi(Z) \cap B_{+}$. Natural dual cones for a WTRO $E$ are defined analogously (a weak ${ }^{*}$ closed cone such that there exists a oneto-one weak* continuous ternary morphism into a $W^{*}$-algebra satisfying $\left.\varphi(\mathfrak{d})=\varphi(Z) \cap B_{+}\right)$. If $Z$ is a WTRO in a $W^{*}$-algebra $M$, then $J(Z)=$ $Z \cap Z^{*} \cap \bar{Z}^{*} Z^{\text {weak* }} \cap \overline{Z Z^{*} \text { weak }^{*}}$, a $W^{*}$-subalgebra of $M$. To see this, note that the latter space is a $W^{*}$-subalgebra, and if $x$ is positive there then $x=\left(x^{*} x\right)^{1 / 2} \in Z^{*} Z$. Similarly, $x \in Z Z^{*}$, and so $x \in J(Z)$.

REMARK. There seems to be no way to reduce the study of cones on TROs to the $*$-TRO case studied in [13]. Clearly if $Z$ is a TRO in a $C^{*}$ algebra $A$, then $W=Z \cap Z^{*}$ is a $*$-TRO, and $W \cap W^{2}=J(Z)$ and $W \cap A_{+}=$ $Z \cap A_{+}$. However, the space $Z \cap Z^{*}$ depends crucially on the particular ambient $C^{*}$-algebra $A$ chosen. That is, if $\theta: Z \rightarrow B$ is a ternary morphism and complete order embedding into another $C^{*}$-algebra $B$, there is no nice relation in general between $W$ and $\theta(Z) \cap \theta(Z)^{*}$.

Corollary 2.2. If $\theta: Z \rightarrow W$ is a ternary morphism between subTROs of $C^{*}$-algebras, and if $\theta$ is positive, then $\theta$ is completely positive.

Proof. Clearly $\theta$ is positive iff $\theta_{\mid J(Z)}$ is positive as a map from $J(Z)$ to $J(W)$. By Lemma 1.1, $\theta_{\mid J(Z)}$ is completely positive. Thus, $\theta_{n}$ is positive as a map from $J\left(M_{n}(Z)\right)=M_{n}(J(Z))$ to $J\left(M_{n}(W)\right)=M_{n}(J(W))$.

Proposition 2.3. Let $Z$ be a TRO, and $u$ a tripotent in $Z^{\prime \prime}$. Then $\mathfrak{d}_{u}$ is a natural cone in $Z$.

Proof. We define two one-to-one ternary morphisms $\theta$ and $\pi$ from $Z$ into $L\left(Z^{\prime \prime}\right)$ as follows:

$$
\theta(z)=\left[\begin{array}{cc}
0 & \left(1-u u^{*}\right) z \\
0 & u^{*} z
\end{array}\right], \quad \pi(z)=\left[\begin{array}{cc}
z u^{*} & z\left(1-u^{*} u\right) \\
0 & 0
\end{array}\right], \quad z \in Z .
$$


Let $T(z)=\theta(z) \oplus \pi(z) \in L\left(Z^{\prime \prime}\right) \oplus^{\infty} L\left(Z^{\prime \prime}\right)$; this is a one-to-one ternary morphism. If $T(z) \geq 0$ then clearly $\left(1-u u^{*}\right) z=z\left(1-u^{*} u\right)=0$ so that $z \in Z_{2}^{\prime \prime}(u)$. Since $u^{*} z \geq 0$ we have $z \in Z \cap \mathfrak{c}_{u}=\mathfrak{d}_{u}$. Conversely, if $z \in \mathfrak{d}_{u}$ it is even easier to see that $T(z) \geq 0$. Thus we have a one-to-one ternary morphism into a $C^{*}$-algebra which is an order embedding.

LEMma 2.4. If $u$ is a tripotent in a TRO $Z$, and $x \in \mathfrak{c}_{u}$, then $u^{*} x=$ $|x| \in Z^{*} Z$.

Proof. We have $u^{*} x \geq 0$ and $u^{*} x u^{*} x=\left(x^{\sharp}\right)^{*} x=x^{*} x$. The result then follows from the uniqueness of square roots.

We recall that $\mathfrak{c}_{u}^{\prime}$ is the weak* closure of $\mathfrak{d}_{u}=\mathfrak{c}_{u} \cap Z$ in $Z^{\prime \prime}$.

Lemma 2.5. For every open tripotent $u \in Z^{\prime \prime}, Z(u)$ is a weak dense $C^{*}$-subalgebra of $Z_{2}^{\prime \prime}(u)$, and is an inner ideal of $Z$. Also, $\mathfrak{c}_{u}^{\prime}=\mathfrak{c}_{u}$. Conversely, every inner ideal of $Z$ which is ternary isomorphic (or equivalently, completely isometrically isomorphic) to a $C^{*}$-algebra arises this way.

Proof. Let $A=Z(u)=\left\{z \in Z: z=u u^{*} z u^{*} u\right\}$, which is an inner ideal. Let $\left(u_{t}\right)$ be a positive net in $A$ converging weak* to $u$. As remarked earlier, $z \mapsto z u^{*}$ is a $*$-homomorphism on $Z_{2}^{\prime \prime}(u)$, and so $u_{t} u^{*} \geq 0$. By a variant of Lemma 2.4, it follows that $u_{t} u^{*} \in Z Z^{*}$. If $z \in A$ then $u_{t} u^{*} z \rightarrow$ $u u^{*} z=z$ weak* ${ }^{*}$. On the other hand, $u_{t} u^{*} \in Z Z^{*}$ as mentioned above, so that $u_{t} u^{*} z \rightarrow z$ weakly in $Z$. Thus convex combinations of $u_{t} u^{*} z$ converge to $z$ in norm. It follows that convex combinations of $u^{*} u_{t} u^{*} z$ converge to $u^{*} z$ in norm, and $u^{*} u_{t} u^{*} z=\left(u_{t}^{\sharp}\right)^{*} z=u_{t}^{*} z \in Z^{*} Z$, so that $u^{*} z \in Z^{*} Z$. Thus $A$ is a subalgebra of $Z_{2}^{\prime \prime}(u)$. To see that it is a $*$-subalgebra, note that a similar argument shows that $u z^{*} \in Z Z^{*}$. Since $z^{*}$ is a norm limit of convex combinations of $z^{*} u u_{t}^{*}$, we see that $u z^{*} u$ is a norm limit of convex combinations of $u z^{*} u u_{t}^{*} u=u z^{*} u_{t}$. The latter is in $Z$ since $u z^{*} \in Z Z^{*}$.

Suppose that $\eta \in Z_{2}^{\prime \prime}(u)$, and $z_{\lambda} \rightarrow \eta$ weak $^{*}$, with $z_{\lambda} \in Z$. Then $u_{s} z_{\lambda}^{*} u_{t}$ is in $A$, since $A$ is an inner ideal. It follows that $u z_{\lambda}^{*} u=z_{\lambda}^{\sharp}$ is in the weak* closure of $A$. Hence also $\eta^{\sharp} \in A^{\perp \perp}$. Thus $A$ is weak* dense in $Z_{2}^{\prime \prime}(u)$, and so $A^{\prime \prime}=Z_{2}^{\prime \prime}(u)$ as von Neumann algebras. Thus if $\eta \in \mathfrak{c}_{u}$ then there is an increasing net in $\mathfrak{d}_{u}$ with weak* limit $\eta$. So $\mathfrak{c}_{u}^{\prime}=\mathfrak{c}_{u}$.

Conversely, assume that $I$ is an inner ideal of $Z$ which is ternary isomorphic to a $C^{*}$-algebra $A$ via a ternary isomorphism $\psi: A \rightarrow I$. Then $\psi^{\prime \prime}: A^{\prime \prime} \rightarrow I^{\prime \prime}=I^{\perp \perp}$ is a one-to-one ternary morphism. If $u=\psi^{\prime \prime}(1)$ then $u$ is a "unitary" tripotent in $I$, in the sense that $I^{\perp \perp}=I_{2}^{\perp \perp}(u)$, and it is easy to see that $I^{\perp \perp}=Z_{2}^{\prime \prime}(u)$ since $I^{\perp \perp}$ is an inner ideal of $Z^{\prime \prime}$. Thus $I=Z(u)$. Moreover, it is clear that $\psi^{\prime \prime}$ is a $*$-isomorphism with respect to the canonical product on $Z_{2}^{\prime \prime}(u)$. Since the identity of $A^{\prime \prime}$ is open, it is evident that $u$ is open. 
We define an inner $C^{*}$-ideal of a TRO $Z$ to be an inner ideal $J$ of $Z$ with a specified positive cone $J_{+}$, which is ternary isomorphic to a $C^{*}$-algebra via an order isomorphism. If, further, $J$ is weak* dense in $Z_{2}^{\prime \prime}(u)$ for a tripotent $u \in Z^{\prime \prime}$ such that $J_{+} \subset \mathfrak{c}_{u}$, then we say that $u$ is a support tripotent for $J$.

LEMma 2.6. An inner $C^{*}$-ideal $J$ of a TRO has a unique support tripotent $u$, which is automatically open, and $J=Z(u)$ and $J_{+}=\mathfrak{d}_{u}$.

Proof. That there exists a support tripotent $u$, which is open, is proved in the last lemma. The proof shows that $J=Z(u)$, and $J_{+}=\mathfrak{d}_{u}$. For the uniqueness of $u$, note that if $v$ were another support tripotent of $J$, then $\mathfrak{c}_{u}=\mathfrak{c}_{u}^{\prime} \subset \mathfrak{c}_{v}$, since $J_{+}=\mathfrak{d}_{u} \subset \mathfrak{c}_{v}$ and the latter set is weak* ${ }^{*}$ closed. By the well known equivalence of (i) and (ii) in the next proposition, $u \leq v$. On the other hand, $v \in Z_{2}^{\prime \prime}(v)=J^{\perp \perp}=Z_{2}^{\prime \prime}(u)$, so that $v=v u^{*} u=u$.

It follows immediately from the last results and discussion earlier in this section that every natural cone on a TRO $Z$ gives rise to an open tripotent, namely the support tripotent of $J(Z)$. Conversely, every open tripotent gives a natural cone by Proposition 2.3.

For the following result, we recall the definition $\widehat{u}=\frac{1}{2}\left(I(u)+I(u)^{2}\right)$ from the introduction.

Proposition 2.7. For tripotents $u, v$ in a TRO $Z$, the following are equivalent:

(i) $u \leq v$ in $Z$.

(ii) $\mathfrak{c}_{u} \subset \mathfrak{c}_{v}$.

(iii) $Z_{2}(u)$ is a $C^{*}$-subalgebra of $Z_{2}(v)$.

(iv) $u \in Z_{2}(v)$ and $u$ is a projection in that $C^{*}$-algebra.

(v) $\widehat{u} \leq \widehat{v}$.

Proof. These are all essentially well known (see e.g. [8]), and easy exercises, except perhaps the equivalence with (v). If $\widehat{u} \leq \widehat{v}$, then $u u^{*} \leq v v^{*}$ and $u^{*} u \leq v^{*} v$. Since $\widehat{u} \widehat{v}=\widehat{u}$, we see that $u / 2=u u^{*} v / 4+u v^{*} v / 4$, and thus $u=u u^{*} v$ and $u \leq v$. The other direction is obtained by multiplying $\widehat{u}$ by $\widehat{v}$ and using the equations $u u^{*} v=v u^{*} u=u$.

COROLlary 2.8. If $u, v$ are open tripotents in the second dual of a TRO $Z$, then the following are equivalent:

(i) $u \leq v$ in $Z^{\prime \prime}$.

(ii) $\mathfrak{d}_{u} \subset \mathfrak{d}_{v}$.

(iii) $Z(u)$ is a $C^{*}$-subalgebra of $Z(v)$.

Also, the correspondence established above between natural cones on $Z$ and open tripotents in $Z^{\prime \prime}$ is bijective, and preserves "order" (ordering cones by inclusion). 
Proof. Suppose that $\mathfrak{d}_{u} \subset \mathfrak{d}_{v}$. Taking weak* closures yields $\mathfrak{c}_{u} \subset \mathfrak{c}_{v}$, so that $u \leq v$ by Proposition 2.7. We leave the other implications as an exercise, using of course that proposition and the earlier established facts summarized in the remark above Proposition 2.7.

REMARKS. 1) Variants of the arguments above show that there is a bijective order preserving correspondence between natural dual cones in a WTRO $E$, and tripotents in $E$.

2) It follows from Corollary 2.8 that maximal natural cones for $Z$ will correspond to maximal open tripotents in $Z^{\prime \prime}$. Maximal open tripotents in $Z^{\prime \prime}$ are studied in the second half of Section 4 below.

Lemma 2.9. Let $Z$ be a TRO, and let $E=Z^{\prime \prime}$, also a TRO in the canonical way. Let $u$ be a tripotent in $E$, so that $\mathfrak{d}_{u}$ is a natural cone by Proposition 2.3. We have:

(1) $\mathfrak{c}_{u}^{\prime} \subset \mathfrak{c}_{u}$.

(2) $\mathfrak{c}_{u}^{\prime}=\mathfrak{c}_{v}$ for an open tripotent $v \in E$ with $v \leq u$.

(3) The closed span $J_{u}$ of $\mathfrak{d}_{u}$ is a $C^{*}$-subalgebra of $E_{2}(u)$, the latter regarded as a $C^{*}$-algebra in the canonical way. Also, $\mathfrak{d}_{u}$ is the positive cone of this $C^{*}$-algebra $J_{u}$.

Proof. Item (1) is obvious, and (2) follows from Proposition 2.7, and Lemmas 2.5 and 2.6. For (3) note that $\mathfrak{d}_{u}=\mathfrak{d}_{v}$, and so $J_{u}$ is a $C^{*}$-subalgebra of $E_{2}(v)$, which in turn is a $C^{*}$-subalgebra of $E_{2}(u)$ by Proposition 2.7(iii).

REMARK. We do not know if it is true that if $Z$ is a TRO in a $C^{*}$-algebra $A$, and if $Z^{\prime \prime}$ is regarded as a TRO in $A^{\prime \prime}$, then $J(Z)$ is weak* dense in $J\left(Z^{\prime \prime}\right)$.

THEOREM 2.10. Let $Z$ be a TRO, set $E=Z^{\prime \prime}$, and let $u$ be a tripotent in $E$. The following are equivalent:

(i) $u$ is an open tripotent (i.e. there is a net $\left(x_{t}\right)$ in $Z$ converging weak ${ }^{*}$ to $u$, satisfying: $u^{*} x_{t} \geq 0, u x_{t}^{*} u=x_{t}$ for all $t$, and $\left(u^{*} x_{t}\right)$ is an increasing net).

(ii) $u \in \mathfrak{c}_{u}^{\prime}$.

(iii) $\widehat{u}=\frac{1}{2}\left(I(u)+I(u)^{2}\right)$ is an open projection in $L(Z)^{\prime \prime}$.

(iv) $\breve{u}=\frac{1}{2}\left(-I(u)+I(u)^{2}\right)$ is an open projection in $L(Z)^{\prime \prime}$.

(v) $u$ is a support tripotent for an inner $C^{*}$-ideal in $Z$.

(vi) $\mathfrak{c}_{u}^{\prime}=\mathfrak{c}_{u}$.

(vii) The closed span $J_{u}$ of $\mathfrak{d}_{u}$ is weak ${ }^{*}$ dense in $E_{2}(u)$.

(viii) $-u$ is an open tripotent.

Proof. Lemmas 2.5 and 2.6 give $(\mathrm{i}) \Leftrightarrow(\mathrm{v})$, and the fact that (i) implies (vi) and (vii). It is easy to see the equivalence of (viii) with (i) from the definition. Clearly (i) implies (ii), and (vi) implies (ii). 
(vii) $\Rightarrow$ (ii). By Lemma 2.9, $J_{u}$ is a $C^{*}$-subalgebra of $E_{2}(u)$. If also $J_{u}^{\perp \perp}=$ $E_{2}(u)$ it follows that $J_{u}=E_{2}(u) \cap Z$, and $E_{2}(u)$ is the "second dual $C^{*}$ algebra" of $J_{u}$. Thus $\left(J_{u}\right)_{+}=\mathfrak{d}_{u}$, and $u \in \mathfrak{c}_{u}^{\prime}$.

(ii) $\Rightarrow$ (iii). Suppose that $\left(x_{t}\right)$ is a net in $\mathfrak{d}_{u}$ converging weak* to $u$. Let

$$
r_{t}=\frac{1}{2}\left[\begin{array}{cc}
x_{t} u^{*} & x_{t} \\
x_{t}^{*} & u^{*} x_{t}
\end{array}\right] .
$$

Since $x_{t}$ is selfadjoint in $Z_{2}^{\prime \prime}(u)$, we have $u^{*} x_{t}=x_{t}^{*} u$ and $x_{t} u^{*}=u x_{t}^{*}$. Thus $r_{t}$ is selfadjoint, and it clearly converges weak* to $\widehat{u}$. In fact $r_{t} \in L(Z)$. Indeed, $x_{t} u^{*} \in Z Z^{*}$ and $u^{*} x_{t} \in Z^{*} Z$ as in Lemma 2.4. Then $\widehat{u}$ is open, since $r_{t} \widehat{u}=r_{t}$.

(iii) $\Rightarrow($ i). Suppose that $\widehat{u}$ is open. Let

$$
r_{t}=\frac{1}{2}\left[\begin{array}{ll}
a_{t} & x_{t} \\
x_{t}^{*} & b_{t}
\end{array}\right]
$$

be a positive net in $L(Z)$ increasing up to $\widehat{u}$. We have $\frac{1}{4} a_{t} u+\frac{1}{4} x_{t} u^{*} u=\frac{1}{2} x_{t}$, and multiplying by $u^{*} u$ yields $x_{t}=a_{t} u$, and $x_{t}=x_{t} u^{*} u$. Similarly $x_{t}=$ $u u^{*} x_{t}$, so that $x_{t} \in Z(u)$. Since $\left(a_{t}\right)$ is positive and increasing, and $x_{t}=a_{t} u$, we find that $\left(x_{t}\right)$ is positive and increasing in $Z_{2}^{\prime \prime}(u)$. Thus $u$ is an open tripotent.

(iii) $\Leftrightarrow($ iv) follows from the equivalence of (viii) with (iii).

REMARK. One may prove directly that (ii) implies (i). Indeed, the proof of Lemma 2.5 shows that $A=Z(u)$ is a $C^{*}$-subalgebra of $Z_{2}^{\prime \prime}(u)$, and $A^{\prime \prime}=Z_{2}^{\prime \prime}(u)$ as von Neumann algebras. It follows that there is an increasing positive net in $A$ with weak* limit $u$. This gives (i).

Corollary 2.11. If $Z$ is a TRO and $u, v$ are tripotents in $Z^{\prime \prime}$, with $v$ open and $u \leq v$, then $u$ is open iff it is open as a projection in $Z_{2}^{\prime \prime}(v)$.

Proof. If $z_{t} \rightarrow u$ weak*, with $\left(z_{t}\right)$ an increasing net in $\mathfrak{d}_{u}$, then $z_{t}$ is an increasing net in $\mathfrak{c}_{v}$. So $u$ is an open projection in $Z_{2}^{\prime \prime}(v)$. Conversely, if $u$ is an open projection in $Z_{2}^{\prime \prime}(v)$, then there is a net $x_{t} \in \mathfrak{d}_{v}$ with $x_{t} \leq u$ in $Z_{2}^{\prime \prime}(v)$, and $x_{t} \rightarrow u$ weak ${ }^{*}$. We have $u v^{*} x_{t}=x_{t}=u u^{*} x_{t}$, and similarly $x_{t} u^{*} u=x_{t}$. Thus $x_{t} \in Z(u)$, and indeed $x_{t} \in \mathfrak{d}_{u}$ since $u^{*} x_{t}=v^{*} x_{t} \geq 0$. Thus $u$ is open by Theorem 2.10(ii).

REMark. The open tripotents in the second dual of a $C^{*}$-algebra that are projections, are exactly the usual open projections [36].

More generally, if $u$ is an open tripotent in $Z^{\prime \prime}$, then it is easy to see from the proof of (iii) $\Rightarrow(\mathrm{i})$ in Theorem 2.10 that $u u^{*}$ and $u^{*} u$ are open projections in $\left(Z Z^{*}\right)^{\prime \prime}$ and $\left(Z^{*} Z\right)^{\prime \prime}$ respectively (cf. [19]).

Proposition 2.12. An increasing net $\left(u_{t}\right)$ of open tripotents in $Z^{\prime \prime}$ has a least upper bound tripotent $u$ in $Z^{\prime \prime}$, namely its weak* limit, and $u$ is also 
open. In terms of cones, $\mathfrak{d}_{u}$ is the norm closure of the union of the cones $\mathfrak{d}_{u_{t}}$. That is, the norm closure of a union of a nested collection of natural cones is a natural cone.

Proof. It is well known [8, Proposition 3.8], and easy to argue directly by a weak* limit argument using separate weak* continuity of the product, that the net has an upper bound tripotent $w$. Working in $Z_{2}^{\prime \prime}(w)$, the $u_{t}$ become an increasing net of projections. Hence they have a supremum projection $u$, which they converge to strongly. It is easy to check that $u$ is also the supremum as a tripotent in $Z^{\prime \prime}$. The spaces $Z_{2}^{\prime \prime}\left(u_{t}\right)$ are $W^{*}$-subalgebras of $Z_{2}^{\prime \prime}(u)$. To see that $u$ is open, by Theorem 2.10 (ii) it suffices to show that $u_{t}$ is in the weak* closure of $\mathfrak{d}_{u}$. But $u_{t}$ is in the weak* closure of $\mathfrak{d}_{u_{t}}$, and

$$
\mathfrak{d}_{u_{t}}=\mathfrak{c}_{u_{t}} \cap Z \subset \mathfrak{c}_{u} \cap Z=\mathfrak{d}_{u} .
$$

Since the $u_{t}$ are open projections in the $C^{*}$-algebra $Z_{2}^{\prime \prime}(u)$ by Corollary 2.11 , the final assertion is essentially well known. We include a proof for completeness. It is clear that $\mathfrak{d}_{u}$ contains the norm closure of the union of the cones $\mathfrak{d}_{u_{t}}$. To get the reverse inclusion, let $A_{t}$ be the $C^{*}$-subalgebra $Z\left(u_{t}\right)$ of $A=Z(u)$. In turn $A$ is a $C^{*}$-subalgebra of $Z_{2}^{\prime \prime}(u)$, and indeed $Z_{2}^{\prime \prime}(u)$ is the second dual $C^{*}$-algebra of $A$. The positive cones of $A_{t}$ and $A$ are $\mathfrak{d}_{u_{t}}$ and $\mathfrak{d}_{u}$ respectively. The weak ${ }^{*}$ closure of $\bigcup_{t} Z_{2}^{\prime \prime}\left(u_{t}\right)$ equals $Z_{2}^{\prime \prime}(u)$, since any $\eta \in Z_{2}^{\prime \prime}(u)$ is the strong limit of $u_{t} u^{*} \eta u^{*} u_{t}$, and we have $u_{t} u^{*} \eta u^{*} u_{t} \in Z_{2}^{\prime \prime}\left(u_{t}\right)$. It follows by basic functional analysis that the norm closure of $\bigcup_{t} A_{t}$ is $A$. From this it is clear that the closure of $\bigcup_{t}\left(A_{t}\right)_{+}$is $A_{+}$.

We leave the following as an exercise.

COROLlary 2.13. The $L^{\infty}$-direct sum $\bigoplus_{i}^{\infty} Z_{i}$ of naturally ordered TROs (resp. dual naturally ordered WTROs), with the obvious cone, is again a naturally ordered TRO (resp. dual naturally ordered WTRO).

3. Further properties of open tripotents. We begin with some facts and lemmas on range tripotents, almost all of which are well known: in the literature (see e.g. [5, 8] and the cited papers of Edwards and Rüttimann, especially [21]) or folklore. However, since the arguments are short and simple we include them here for the reader's convenience. Later in this section we establish the basic "calculus" of open tripotents, following (and freely using ideas from) the basic calculus of open projections, and the calculus of tripotents established in the aforementioned papers. In view of the bijective correspondence from Corollary 2.8, this calculus may be reread as constituting most of the basic "theory of natural cones". We will not usually explicitly state the cone version of each result below, but leave this to the reader. 
Let $E$ be a WTRO. For each $x \in E$, we consider the range tripotent $r(x)$ in $E$. This is the tripotent in $E$ with the property that $x=r(x)|x|$ and $r(x)^{*} r(x)$ is the support projection of $|x|$ (namely, $r(x)$ is the partial isometry in the polar decomposition of $x$, see e.g. 8.5.22 in [12]). Such a tripotent is unique: it is the smallest tripotent $u$ in $E$ with the property that $x=u|x|$. (To see this, note that if $x=u|x|$, then $u r(x)^{*} r(x)|x|=u|x|=x=r(x)|x|$, and so $\operatorname{ur}(x)^{*} r(x)=r(x)$.) We have

$$
x r(x)^{*} r(x)=r(x)|x| r(x)^{*} r(x)=r(x)|x|=x,
$$

since $1-r(x)^{*} r(x)$ is the projection onto $\operatorname{Ran}(|x|)^{\perp}=\operatorname{Ker}(|x|)$. Also,

$$
|x| r(x)^{*} r(x)=\left(r(x)^{*} r(x)|x|\right)^{*}=|x|,
$$

and so

$$
r(x) x^{*} r(x)=r(x)|x| r(x)^{*} r(x)=r(x)|x|=x .
$$

This shows that $x \in Z^{\prime \prime}(r(x))_{+}$, since $r(x)^{*} x=|x| \geq 0$.

Define $C_{0}(x)$ to be the norm closure of the span of odd polynomials of $x$. By [19, Lemma 3.2], $C_{0}(x)$ is a commutative $C^{*}$-subalgebra of $Z_{2}^{\prime \prime}(r(x))$, and odd polynomials in $x$ are the same in either product. It follows easily that $C_{0}(x)$ is ternary isomorphic to $C_{0}(\operatorname{Sp}(x))$ where the spectrum is taken in $Z_{2}^{\prime \prime}(r(x))$. Thus the elements in $C_{0}(x)$ coincide with the usual functional calculus in the $W^{*}$-algebra $Z_{2}^{\prime \prime}(r(x))$. If $Z$ is a $*$-TRO and if $x \in Z_{\mathrm{sa}}$, then clearly $r(x)$ is selfadjoint.

LEMMA 3.1. If $u$ is a tripotent in a WTRO $Z$, then $\mathfrak{c}_{u}=\{z \in Z: r(z) \leq u\}$. If $Z$ is a TRO and $u \in Z^{\prime \prime}$, then $\mathfrak{d}_{u}=\{z \in Z: r(z) \leq u\}$.

Proof. If $r(z) \leq u$ then $z \in \mathfrak{c}_{r(z)} \subset \mathfrak{c}_{u}$, by Proposition 2.7. Conversely, if $z \in \mathfrak{c}_{u}$, then $u|z|=u u^{*} z=z$ by Lemma 2.4, so that $r(z) \leq u$. The final assertion follows immediately from the first one.

LEMma 3.2. If $u$ is a tripotent in a WTRO E, and if $x \in \mathfrak{c}_{u}$, then $r(x)$ is the support projection of $x$ in the $W^{*}$-algebra $E_{2}(u)$.

Proof. Since $x \in \mathfrak{c}_{u}$ we have $r(x) \leq u$ by Lemma 3.1, and so $r(x)$ is a projection in $E_{2}(u)$. We have $r(x) u^{*} x=r(x) r(x)^{*} x=x$. If $v$ is another projection in $E_{2}(u)$ with $v u^{*} x=x$ then $v u^{*} r(x)|x|=r(x)|x|$. This implies that $v u^{*} r(x)=r(x)$ so that $v \geq r(x)$. Thus $r(x)$ is the support projection of $x$ in $E_{2}(u)$.

Lemma 3.3. Let $Z$ be a TRO. The range tripotent $r(x)$ of any $x \in Z$ coincides with the weak* limit of $x^{1 /(2 n-1)}$, the power taken in the $W^{*}$-algebra $Z_{2}^{\prime \prime}(r(x))$, and is open. Furthermore, $Z(r(x))$ is the smallest inner ideal in $Z$ containing $x$.

Proof. We work in the $W^{*}$-algebra $Z_{2}^{\prime \prime}(r(x))$. As we said earlier, $x \geq 0$ in $Z_{2}^{\prime \prime}(r(x))$. The first statement then follows from Lemma 3.2, and well 
known properties of support projections in a $W^{*}$-algebra. As mentioned at the beginning of this section, odd polynomials of $x$ are the same in either of the two products, and lie in $Z$. Since $x^{1 /(2 n-1)}$ is a norm limit of odd polynomials of $x$ in $Z_{2}^{\prime \prime}(r(x))$, we see that $x^{1 /(2 n-1)}$ lies in $Z$. Thus $r(x)$ is open by (ii) of Theorem 2.10. For the last statement, it is clear that any weak* closed inner ideal of $Z^{\prime \prime}$ containing $x$ must contain $r(x)$, and thus all of $Z_{2}^{\prime \prime}(r(x))$ by definition of inner ideal. Hence, any inner ideal $I$ of $Z$ must contain $Z(r(x))$ since $\bar{I}^{\text {weak* }}$ is an inner ideal of $Z^{\prime \prime}$ (see also see Lemma 3.7 of [19]).

In the last proof we showed that if $x \in \operatorname{Ball}(Z)$, then $r(x)$ is an increasing weak ${ }^{*}$ limit of powers $x^{1 /(2 n-1)}$, which in turn are norm limits of odd polynomials in $x$. This is also true if $x \in E$ for a WTRO $E$, with the weak* limit being in the weak* topology of $E$. We will use these facts frequently below, often silently.


ing net of range tripotents.

Proof. A tripotent which is a limit of an increasing net of range tripotents is open by Proposition 2.12 and Lemma 3.3. Conversely, suppose that $u$ is open. Then $u$ is a weak* limit of an increasing net $\left(z_{t}\right)$ in $Z(u)$. By Lemma $3.2, r\left(z_{t}\right)$ is the support projection of $z_{t}$ in the $W^{*}$-algebra $Z_{2}^{\prime \prime}(u)$. Thus, the net $\left(r\left(z_{t}\right)\right)$ of range tripotents is increasing, and we have $z_{t} \leq r\left(z_{t}\right) \leq u$. It follows that $r\left(z_{t}\right) \rightarrow u$ weak*

REMARK. One may also construct "open spectral tripotents" as follows. If $x$ is an element in a TRO $Z$, then $x$ is positive in the $W^{*}$-algebra $Z_{2}^{\prime \prime}(r(x))$. If $U$ is any open set in $\operatorname{Sp}(x) \subset[0, \infty)$, then the spectral projection $\chi_{U}(x)$ in the $W^{*}$-algebra $Z_{2}^{\prime \prime}(r(x))$ is open, and hence it is an open tripotent in $Z^{\prime \prime}$. A special case of course is if we take $U=(0, \infty)$; then the associated open spectral tripotent $u$ is just $r(x)$. Indeed, this is a well known formula for a support projection in a $W^{*}$-algebra.

We now turn to properties of general open tripotents.

Proposition 3.5. If $\theta: Z \rightarrow W$ is a ternary morphism between TROs, and if $u$ is an open tripotent in $Z^{\prime \prime}$, then $v=\theta^{\prime \prime}(u)$ is an open tripotent in $W^{\prime \prime}$. Also, $\theta$ restricts to a $*$-homomorphism $Z(u) \rightarrow Z(v)$, and $\theta\left(\mathfrak{d}_{u}\right) \subset \mathfrak{d}_{v}$. If also $\theta$ is surjective, then $\theta\left(\mathfrak{d}_{u}\right)=\mathfrak{d}_{v}$.

Proof. Suppose that $z_{t} \in Z$ with $u^{*} z_{t} \geq 0, z_{t}=u z_{t}^{*} u$, and $z_{t} \rightarrow u$ weak* ${ }^{*}$. Applying $\theta$ we obtain a net $\left(\theta\left(z_{t}\right)\right)$ with analogous properties, so that $v$ is open by Theorem 2.10(ii). Clearly $\theta^{\prime \prime}$ is a $*$-homomorphism $Z_{2}^{\prime \prime}(u) \rightarrow Z_{2}^{\prime \prime}(v)$, and thus restricts to a $*$-homomorphism $Z(u) \rightarrow Z(v)$. If also $\theta$ is surjective, 
then $\theta^{\prime \prime}\left(Z_{2}^{\prime \prime}(u)\right)$ is an inner ideal containing $v$, so that $\theta^{\prime \prime}\left(Z_{2}^{\prime \prime}(u)\right)=Z_{2}^{\prime \prime}(v)$. From this it follows that $\theta(Z(u))=Z(v)$ and $\theta\left(\mathfrak{d}_{u}\right)=\mathfrak{d}_{v}$.

REMARK. Thus $\theta$ will be completely positive on $Z$ with its ordering determined by $u$.

LEMMA 3.6. If $r$ is an antisymmetric projection in $\bar{L}(E)$ for a WTRO $E$, then $r=\widehat{v}$ for a tripotent $v \in E$.

Proof. Suppose that $r=a \otimes e_{11}+b \otimes e_{12}+b^{*} \otimes e_{21}+c \otimes e_{22}$. Squaring $r$ yields $a^{2}+b b^{*}=a$. Antisymmetry yields $a^{2}-b b^{*}=0$. Thus $2 a^{2}=a$. Hence $2 a$ is a projection. Since $(2 b)(2 b)^{*}=4 a^{2}=2 a$, we see $2 b$ is a partial isometry. Similarly $(2 b)^{*}(2 b)=2 c$. The result follows.

We now turn to the supremum $u \vee v$ of two tripotents. Most of the following result is in [5], but for convenience we give quick proofs.

Lemma 3.7. Suppose $u, v$ are tripotents in a WTRO E. The following are equivalent:

(i) $u \vee v$ exists.

(ii) $\widehat{u} \perp \breve{v}$.

(iii) $u v^{*} v=u u^{*} v$ and $v v^{*} u=v u^{*} u$.

(iv) $\{u, v\}$ is dominated by a tripotent $w \in E$.

In this case, $\widehat{u \vee v}=r(\widehat{u}+\widehat{v})=\widehat{u} \vee \widehat{v}$ and $u \vee v=r(u+v)$.

Proof. (i) $\Rightarrow$ (iv). Obvious.

(iv) $\Rightarrow$ (iii). Suppose that $u \leq w$ and $v \leq w$. We have

$$
u v^{*} v=u w^{*} w v^{*} v=u w^{*} v=u u^{*} u w^{*} v=u u^{*} v .
$$

The proof of the other statement is similar.

(iii) $\Rightarrow$ (ii). Clear from direct multiplication, noting that $u u^{*} v v^{*}=u v^{*}$ and $u^{*} v=u^{*} u v^{*} v$.

(ii) $\Rightarrow($ i $)$. Multiplication shows that $(\widehat{u}+\widehat{v}) \Theta(\widehat{u}+\widehat{v})=0$. Consequently, $p(\widehat{u}+\widehat{v}) \Theta(q(\widehat{u}+\widehat{v}))=0$ for any odd polynomials $p$ and $q$. Taking weak ${ }^{*}$ limits, it follows that $r(\widehat{u}+\widehat{v}) \Theta(r(\widehat{u}+\widehat{v}))=0$, and so $r(\widehat{u}+\widehat{v})$ is an antisymmetric projection, and thus equals $\widehat{w}$ for a tripotent $w$ by Lemma 3.6. As is well known, $\widehat{u} \vee \widehat{v}=r(\widehat{u}+\widehat{v})$, so that $\widehat{u} \vee \widehat{v}=\widehat{w}$. By Proposition 2.7 we have $u \leq w$ and $v \leq w$, and so $u \vee v \leq w$. If $w_{0}=u \vee v$ then $\widehat{w}_{0} \geq \widehat{u}$ and $\widehat{w}_{0} \geq \widehat{v}$, so that $\widehat{w}_{0} \geq \widehat{u} \vee \widehat{v}=\widehat{w}$. Proposition 2.7 gives $w_{0} \geq w$, so that $w=u \vee v$. Finally, $r(u+v)=u \vee v$ by [8, Proposition 3.9(i)].

Proposition 3.8. Let $Z$ be a TRO. A family $\left\{u_{i}: i \in I\right\}$ of open tripotents in $Z^{\prime \prime}$, which are bounded above by a tripotent in $Z^{\prime \prime}$, has a least upper bound amongst the tripotents in $Z^{\prime \prime}$, and this is an open tripotent.

Proof. We first show that if $u, v$ are open tripotents in $Z^{\prime \prime}$, which are bounded above by a tripotent, then the sup tripotent $w=u \vee v$ (which 
exists by the previous lemma) is open. By Lemma 2.9 and its proof, $\operatorname{Span}\left(\mathfrak{d}_{w}\right)$ $=Z(e)$ for an open tripotent $e \leq w$ with $\mathfrak{d}_{w}=\mathfrak{d}_{e}$. Since $\mathfrak{d}_{u} \subset \mathfrak{d}_{w}=\mathfrak{d}_{e}$, we have $u \leq e$. Similarly $v \leq e$, and so $e=w$.

It now follows by induction that the supremum of any finite family of open tripotents which are bounded above by a tripotent, is open. The result then follows easily from Proposition 2.12.

We say that tripotents $u$ and $v$ commute if $v^{*} u=u^{*} v$ and $v u^{*}=u v^{*}$ (Harris calls this $*$-commuting). We write $u \perp v$ if these quantities are zero.

Corollary 3.9. Let $Z$ be a TRO, and let $u, v$ be two commuting open tripotents satisfying $v u^{*} u=v v^{*} u$. The supremum $u \vee v$ in the set of tripotents in $Z^{\prime \prime}$ exists, is open, and is given by the formula $u+v-v v^{*} u$. In particular, $u \vee v=u+v$ is open if $u \perp v$.

Proof. Let $u, v$ be as stated. Then, by Lemma 3.7, $w=u \vee v$ exists, and it is open by Proposition 3.8. By commutativity, $u w^{*} v=u u^{*} v=v u^{*} u=v w^{*} u$, so $u$ and $v$ commute as projections in $Z_{2}(w)$. As is well known in this case, $w=u \vee v=u+v-u w^{*} v=u+v-u u^{*} v$. The result follows.

REMARK. We do not know how to describe cones corresponding to suprema of tripotents, even if they are orthogonal. For example, the usual cone in $M_{2}$ seems not nicely related to $\mathfrak{d}_{E_{11}}$ and $\mathfrak{d}_{E_{22}}$.

Corollary 3.10. Let $Z$ be a TRO. The infimum $u \wedge v$ of two commuting open tripotents $u$ and $v$ in $Z^{\prime \prime}$ is open, and is given by the formula $\frac{1}{2}\left(v v^{*} u+\right.$ $\left.v u^{*} u\right)$.

Proof. Let $u, v$ be two commuting open tripotents. Then $\widehat{u}$ and $\widehat{v}$ are two commuting open projections in $L(Z)^{\prime \prime}$. It is well known that the infimum of two commuting open projections is open $[1,2]$, and so $\widehat{u} \widehat{v}$ is open. But $\widehat{u v}=\widehat{w}$, where $w=\frac{1}{2}\left(v v^{*} u+v u^{*} u\right)$. Since $w$ is a tripotent, $w$ is open by Theorem 2.10. It is easy to check that $w=u \wedge v$ (or see e.g. [5]).

The infimum of a collection of open tripotents amongst all tripotents in $Z^{\prime \prime}$ need not be open. However, there is an infimum amongst the open tripotents in $Z^{\prime \prime}$ :

Lemma 3.11. Suppose that $Z$ is a TRO and that $\mathcal{F}=\left\{u_{\alpha}: \alpha \in I\right\}$ is a collection of open tripotents in $Z^{\prime \prime}$. Then there exists an infimum $u$ for $\mathcal{F}$ amongst the open tripotents in $Z^{\prime \prime}$. Also, $\mathfrak{d}_{u}=\bigcap_{\alpha \in I} \mathfrak{d}_{u_{\alpha}}$.

Proof. Clearly the infimum is the supremum of the open tripotents $v$ such that $v \leq u_{\alpha}$ for every $\alpha \in I$. This is open by Proposition 3.8. Since $u \leq u_{\alpha}$ for every $\alpha \in I$, we have $\mathfrak{c}_{u} \subset \mathfrak{c}_{u_{\alpha}}$, and so $\mathfrak{c}_{u} \subset \bigcap_{\alpha \in I} \mathfrak{c}_{u_{\alpha}}$. Conversely, if $x \in \bigcap_{\alpha \in I} \mathfrak{c}_{u_{\alpha}}$ then by Lemma 3.1 we have $r(x) \leq u_{\alpha}$ for every $\alpha \in I$, and so $r(x) \leq u$. By Lemma 3.1 again, $x \in \mathfrak{c}_{u}$. Thus $\mathfrak{c}_{u}=\bigcap_{\alpha \in I} \mathfrak{c}_{u_{\alpha}}$, and the result is now obvious. 
The lemma asserts that an intersection of natural cones is a natural cone. This is valid at the matrix level too, and it will play a role later. That is, $\left(\mathfrak{d}_{u}\right)_{n}=\bigcap_{\alpha}\left(\mathfrak{d}_{u_{\alpha}}\right)_{n}$ for all $n \in \mathbb{N}$. A direct proof of this: if $\left\{\mathfrak{c}_{\alpha}\right\}$ is a family of natural dual cones in a WTRO $E$, and if $T_{\alpha}: E \rightarrow B_{\alpha}$ is a ternary morphism which is an order embedding for the cone $\mathfrak{c}_{\alpha}$, then the map $T: Z \rightarrow \bigoplus_{\alpha}^{\infty} B_{\alpha}$ taking $z \in Z$ to $\bigoplus_{\alpha} T_{\alpha}(z)$ is a ternary morphism which is an order embedding for the cone $\mathfrak{c}=\bigcap_{\alpha} \mathfrak{c}_{\alpha}$. Hence it is a complete order embedding by Corollary 2.2. Thus if $\left[z_{i j}\right] \in M_{n}(E)$ then $\left[z_{i j}\right] \in \mathfrak{c}_{n}$ iff $\left[T\left(z_{i j}\right)\right] \geq 0$ iff $\left[T_{\alpha}\left(z_{i j}\right)\right] \geq 0$ for each $\alpha$. In turn, this happens iff $\left[z_{i j}\right] \in\left(\mathfrak{c}_{\alpha}\right)_{n}$ for each $\alpha$, that is, iff $\left[z_{i j}\right] \in \bigcap_{\alpha}\left(\mathfrak{c}_{\alpha}\right)_{n}$. Thus $\mathfrak{c}_{n}=\bigcap_{\alpha}\left(\mathfrak{c}_{\alpha}\right)_{n}$. In terms of tripotents,

$$
\left(\bigwedge_{\alpha} u_{\alpha}\right) \otimes I_{n}=\bigwedge_{\alpha}\left(u_{\alpha} \otimes I_{n}\right) .
$$

The same argument works for natural cones in a TRO $Z$, or this can be deduced from the above by taking $E=Z^{\prime \prime}$.

Corollary 3.12. Let $n \in \mathbb{N}$. The map $z \mapsto z \otimes I_{n}$ from a WTRO $E$ into $M_{n}(E)$ is a one-to-one ternary morphism that preserves infima of tripotents, and suprema of tripotents where they exist.

Proof. The statement about infima is demonstrated above. Next, if $\left\{u_{\alpha}\right\}$ is a family of tripotents in $E$ which are bounded above by a tripotent $u$, then $u_{\alpha} \otimes I_{n} \leq u \otimes I_{n}$, so that $\left\{u_{\alpha} \otimes I_{n}\right\}$ is bounded above by $u \otimes I_{n}$, where $u=\bigvee_{\alpha} u_{\alpha}$. Conversely, suppose that $\left\{u_{\alpha} \otimes I_{n}\right\}$ is bounded above by a tripotent $w \in M_{n}(E)$. It is easy to see that $w_{i j} u_{\alpha}^{*} u_{\alpha}=u_{\alpha}$ for each $i, j, \alpha$. Thus $u\left(w_{i j}\right) \geq u_{\alpha}$. Hence $\left\{u_{\alpha}\right\}$ is bounded above by $v=\bigwedge_{i, j} u\left(w_{i j}\right)$, so that $u \leq v$ where $u=\bigvee_{\alpha} u_{\alpha}$. Note that

$$
w_{i j} u^{*} u=w_{i j} u\left(w_{i j}\right)^{*} u\left(w_{i j}\right) u^{*} u=u\left(w_{i j}\right) u^{*} u=u .
$$

Thus $u_{\alpha} \otimes I_{n} \leq u \otimes I_{n} \leq w$. Hence the supremum of $\left\{u_{\alpha} \otimes I_{n}\right\}$ amongst the tripotents in $M_{n}(E)$ is $u \otimes I_{n}$.

We will need a few matrix tricks which we have not seen in the literature. For $x \in \operatorname{Ball}(Z)$ define

$$
\widehat{x}=\frac{1}{2}\left[\begin{array}{cc}
\left|x^{*}\right| & x \\
x^{*} & |x|
\end{array}\right] \in L(Z) .
$$

Writing this as a sum of a diagonal matrix and an off-diagonal matrix, we see that $\|\widehat{x}\| \leq 1$. Letting

$$
z=\frac{1}{\sqrt{2}}\left[\begin{array}{cc}
0 & r(x)|x|^{1 / 2} \\
0 & |x|^{1 / 2}
\end{array}\right],
$$

we have $z z^{*}=\widehat{x}$. Thus $\widehat{x}$ is positive. 
LEMMA 3.13. If $Z$ is a WTRO and $y \in \bar{L}(Z)$ then $y=\widehat{x}$ for an $x \in$ $\operatorname{Ball}(Z)$ iff $0 \leq y \leq \widehat{u}$ for a tripotent $u \in Z$. If these hold then $x \in \mathfrak{c}_{u}$.

Proof. If $y=\widehat{x}$ then matrix multiplication shows that $0 \leq y \leq \widehat{r(x)}$. For the converse, if $0 \leq y \leq \widehat{u}$ then multiplying $y$ with $\widehat{u}$, we find that $\frac{1}{2} u u^{*} y_{11}+\frac{1}{2} u y_{12}^{*}=y_{11}$ and $\frac{1}{2} u^{*} y_{11}+\frac{1}{2} u^{*} u y_{12}^{*}=y_{12}^{*}$. Since $y_{11} \leq \frac{1}{2} u u^{*}$, it follows that $u y_{12}^{*}=y_{11}=y_{12} u^{*}$ and $u^{*} y_{11}=u^{*} u y_{12}^{*}=y_{12}^{*}$. Thus it is easy to see that $y_{11}^{2}=y_{12} y_{12}^{*}$. A similar argument shows that $y_{22}^{2}=y_{12}^{*} y_{12}$. The equalities above also show that $y_{12} \in Z_{2}(u)$, and since $y_{12} u^{*}=y_{11} \geq 0$, it follows that $y_{12}$ lies in $\mathfrak{c}_{u}$.

COROLlary 3.14. If $\left\{u_{i}\right\}$ is an increasing net of tripotents in a WTRO $Z$ then $u_{i} \rightarrow u$ weak ${ }^{*}$ iff $\widehat{u}_{i} \rightarrow \widehat{u}$ weak ${ }^{*}$.

Proof. If $u_{i} \rightarrow u$ weak ${ }^{*}$, then $\left\{\widehat{u}_{i}\right\}$ is an increasing net of projections dominated by $\widehat{u}$. Its weak* limit, by Lemma 3.13, equals $\widehat{v}$ for some $v$. Looking at convergence in the 1-2 corner, we see $u=v$. So $\widehat{u}_{i} \rightarrow \widehat{u}$ weak* $^{*}$. The other direction is easier.

Corollary 3.15. Let $\left\{u_{\lambda}\right\}$ be a family of tripotents that pairwise satisfy any one of the conditions in Lemma 3.7. Then $\widehat{\bigvee_{\lambda} u_{\lambda}}=\bigvee_{\lambda} \widehat{u}_{\lambda}$. If also the $u_{\lambda}$ are all open, then so is $\bigvee_{\lambda} u_{\lambda}$.

Proof. If $\widehat{v} \perp \breve{u}, \widehat{w} \perp \breve{u}$, and $\widehat{v} \perp \breve{w}$, then by Lemma 3.7 we have $\widehat{v \vee w}=$ $r(\widehat{v}+\widehat{w})$. Since $p(\widehat{v}+\widehat{w}) \perp \breve{u}$ for any odd polynomial $p$, we have $\breve{u} \perp r(\widehat{v}+\widehat{w})=$ $\widehat{v \vee w}$. By Lemma 3.7, $u \vee(v \vee w)$ exists. By induction, $\bigvee_{\lambda \in F} u_{\lambda}$ exists for any finite set $F$, and $\widehat{\bigvee_{F} u_{\lambda}}=\bigvee_{F} \widehat{u}_{\lambda}$. We leave the rest as an exercise, using the last corollary.

The material in the rest of this section is used in [9].

If $Z$ is a TRO and $x \in \operatorname{Ball}(Z)$, then Edwards and Rüttimann define $u(x)$ to be the weak* limit in $Z^{\prime \prime}$, or equivalently in the $W^{*}$-algebra $Z_{2}^{\prime \prime}(r(x))$, of $x^{2 n+1}$, where $x^{2 n+1}=x x^{*} x \cdots x^{*} x$, a product of $2 n+1$ terms (see [17, Lemma 3.4]).

Definition 3.16. If $Z$ is a TRO then a tripotent $v$ in $Z^{\prime \prime}$ is compact if it is the weak* limit of a decreasing net of tripotents $u\left(x_{\lambda}\right)$, where each $x_{\lambda} \in \operatorname{Ball}(Z)$.

REmarks. (1) We do not need this here, but it is an easy exercise to show that $u(x)$ is the largest tripotent $v$ such that $v=v x^{*} v$ (see also [17, Lemma 3.4]).

(2) Clearly $u(x)$ is compact for any $x \in \operatorname{Ball}(Z)$.

(3) If $Z$ is a $C^{*}$-algebra $A$, and $x \in A_{+}$, then $u(x)$ is a projection. Note that in this case, if $0 \leq x \leq y \leq 1$ then $u(x) \leq x \leq y$, so that $u(x) \leq y^{n}$ for any $n \in \mathbb{N}$. Thus $u(x) \leq u(y)$. 
(4) It is essentially implicit in the main result from [17] that if $Z$ is a WTRO, and $x, y \in \operatorname{Ball}(Z)$, then $u(x) \wedge u(y)=u((x+y) / 2)$. This is used in $[9]$.

Lemma 3.17. If $Z$ is a TRO and $x \in \operatorname{Ball}(Z)$ then $u(\widehat{x})=\widehat{u(x)}$ and $r(\widehat{x})=\widehat{r(x)}$.

Proof. A simple computation shows that

$$
\widehat{x}^{2 n+1}=\frac{1}{2}\left[\begin{array}{cc}
\left|x^{*}\right|^{2 n+1} & x^{2 n+1} \\
\left(x^{*}\right)^{2 n+1} & |x|^{2 n+1}
\end{array}\right],
$$

where $x^{2 n+1}$ is as above Definition 3.16. The weak* limit of $\widehat{x}^{2 n+1}$ is a projection $q$ say, whose 1-2 entry is $\frac{1}{2} u(x)$ by the last displayed formula. As we said earlier, $\widehat{x} \leq \widehat{r(x)}$. This, together with Lemma 3.13, shows that $q=\widehat{u(x)}$. That is, $u(\widehat{x})=\widehat{u(x)}$. If $p$ is an "odd polynomial", then

$$
p(\widehat{x})=\frac{1}{2}\left[\begin{array}{cc}
p\left(\left|x^{*}\right|\right) & p(x) \\
p\left(x^{*}\right) & p(|x|)
\end{array}\right] .
$$

It follows by a norm approximation that the same relation holds with $p$ replaced by the function $t^{1 /(2 n+1)}$. All of these quantities are bounded above by $\widehat{r(x)}$. In the weak* limit, and using Lemma 3.13 , it follows that $r(\widehat{x})$ $=\widehat{r(x)}$.

Let $v$ be a tripotent in $Z^{\prime \prime}$, for a TRO $Z$. Following [5], we say that $v$ belongs locally to $Z$ if $v^{*} v$ is a closed projection in $\left(Z^{*} Z\right)^{\prime \prime}$ and $v=x v^{*} v$ for an element $x \in \operatorname{Ball}(Z)$. The following known result $[5,21]$, which we give a quick alternative proof of for the reader's convenience, shows that this is equivalent to $v$ being compact:

Proposition 3.18 (Akemann-Pedersen, Edwards and Rüttimann). Let $u$ be a tripotent in $Z^{\prime \prime}$ for a TRO $Z$. The following are equivalent:

(i) $u$ is compact.

(ii) $\widehat{u}$ is a compact projection in $L(Z)^{\prime \prime}$ (that is, there exists a decreasing net in $L(Z)$ converging weak ${ }^{*}$ to $\left.\widehat{u}\right)$.

(iii) $u$ belongs locally to $Z$.

Also, a weak $k^{*}$ limit of a decreasing net of compact tripotents is compact.

Proof. (i) $\Rightarrow$ (ii). There is a family of norm one elements $x_{\lambda}$ such that $u\left(x_{\lambda}\right)$ is a decreasing net of tripotents converging weak* to $u$. Then $\widehat{u\left(x_{\lambda}\right)}$ is a decreasing net of projections converging weak ${ }^{*}$ to a projection $p \geq \widehat{u}$, say. By Lemma 3.13, we have $p=\widehat{x}$ for some $x \in Z^{\prime \prime}$, and looking at the 1-2 entry we see that $x=u$. So $\widehat{u\left(x_{\lambda}\right)} \rightarrow \widehat{u}$ weak $^{*}$. By Lemma 3.17, we have $\widehat{u\left(x_{\lambda}\right)}=u(a)$ for some $a \in L(Z)$, and is thus a compact projection. Thus $\widehat{u}$ 
is closed, being a decreasing limit of closed projections. Since it is bounded above by an element in $L(Z)$, it is compact.

(ii) $\Leftrightarrow$ (iii). This is Proposition 4.9 of [5].

(iii) $\Rightarrow$ (i). If $u=x u^{*} u$ for an element $x \in \operatorname{Ball}(Z)$, then $u=u u^{*} x=$ $u u^{*} x u^{*} u$ (see [5, Lemma 4.8]), which implies that

$$
x=u u^{*} x u^{*} u+\left(1-u u^{*}\right) x\left(1-u^{*} u\right)=u+\left(1-u u^{*}\right) x\left(1-u^{*} u\right) .
$$

It follows that $x^{2 n+1}=u+\left(1-u u^{*}\right) x^{2 n+1}\left(1-u^{*} u\right)$, where $x^{2 n+1}$ as usual means $x x^{*} x \cdots x^{*} x$, a product of $2 n+1$ terms. This implies that $r(x) \geq u$. Since $r(x)^{*} r(x) \geq u^{*} u$, by the Urysohn lemma for $C^{*}$-algebras there is a decreasing net $\left(y_{\lambda}\right)$ in $Z^{*} Z$ converging to $u^{*} u$ with $y_{\lambda} \leq r(x)^{*} r(x)$. Now $r(x) y_{\lambda}$ lies in $Z_{2}^{\prime \prime}(r(x))$, and so $\left(r(x) y_{\lambda}\right)$ is decreasing in $Z_{2}^{\prime \prime}(r(x))$. Hence $x\left(r(x) y_{\lambda}\right)^{*} x$ is a decreasing net in $Z(r(x))_{+} \cap \operatorname{Ball}(Z)$ converging weak ${ }^{*}$ to $x u^{*} u r(x)^{*} x=u u^{*} x=u$. Thus the projections $u\left(x\left(r(x) y_{\lambda}\right)^{*} x\right)$ are a decreasing (by Remark (3) after Definition 3.16) net converging weak* to $u$.

Finally, given a decreasing net of compact tripotents with limit $u$, a slight modification of the first paragraph of the proof shows that $\widehat{u}$ is compact.

We now give an Urysohn lemma for TROs, based on Akemann's Urysohn lemma for $C^{*}$-algebras $[1,2,3,5]$. See also [23] for a related result, with a different proof strategy (which relies on results of the second author [33]).

TheOREM 3.19. Suppose that $Z$ is a TRO and that $v$ and $u$ are tripotents in $Z^{\prime \prime}$ such that $v$ is compact, $u$ is open, and $v \leq u$. Then there exists an element $x \in Z$ such that $v \leq x \leq u$ in the $W^{*}$-algebra $Z_{2}^{\prime \prime}(u)$.

Proof. By Proposition 2.7 we have $\widehat{v} \leq \widehat{u}$. By the Urysohn lemma for $C^{*}$-algebras, there is an element $y \in L(Z)+$ such that $\widehat{v} \leq y \leq \widehat{u}$. By Lemma 3.13, we have $y=\widehat{x}$ for some $x \in \mathfrak{c}_{u} \subset Z^{\prime \prime}$. By Lemma 3.17 it follows that $u(y)=\widehat{u(x)}$ and $r(y)=\widehat{r(x)}$. By Proposition 2.7 we have $v \leq u(x) \leq r(x) \leq u$. Hence $v \leq x \leq u$.

REMARKS. (1) It is easy to see from this Urysohn lemma that if $v \leq u$ are tripotents in $Z^{\prime \prime}$ with $u$ open, then $v$ is compact in $Z^{\prime \prime}$ iff $v$ is compact as a projection in $Z(u)^{\prime \prime}=Z_{2}^{\prime \prime}(u)$.

(2) There are also regularity properties for open and compact tripotents, analogous to the case of projections in a $C^{*}$-algebra (see e.g. Akemann's regularity property described in [27, Section 2]). Theorem 3.20 below corresponds to the normality separation property one has in locally compact topological spaces.

The following variant of Urysohn's lemma solves an open problem from [23], in the special case of TROs (see [23, Problem 2.13]).

THEOREM 3.20. Suppose that $Z$ is a TRO and that $v$ and $w$ are compact tripotents in $Z^{\prime \prime}$ with $v^{*} w=v w^{*}=0$. Then there exist elements $x, y \in$ 
$\operatorname{Ball}(Z)$ such that $r(x)^{*} r(y)=r(x) r(y)^{*}=0$, and $v \leq x$ and $w \leq y$ in the $C^{*}$-algebras $Z_{2}^{\prime \prime}(r(x))$ and $Z_{2}^{\prime \prime}(r(y))$ respectively.

Proof. Clearly $\widehat{v} \perp \widehat{w}$ are compact, and so $\widehat{v+w}=\widehat{v}+\widehat{w}$ is a closed projection. Since both $\widehat{v}$ and $\widehat{w}$ are dominated by an element in $L(Z)_{+}$, so is $\widehat{v}+\widehat{w}$. Hence $\widehat{v+w}$ is a compact projection [5], and so $v+w$ is a compact tripotent. Thus there exists an open tripotent $u \geq v+w$. Working inside $Z_{2}^{\prime \prime}(u)$, we see that $v, w$ are compact mutually orthogonal projections (see Remark (1) above), and by [4, Proposition 2.6] there exist mutually orthogonal open projections $p, q$ in $Z_{2}^{\prime \prime}(u)$ with $v \leq p, w \leq q$. By the noncommutative Urysohn lemma, there exist elements $x, y \in \operatorname{Ball}(Z) \cap Z_{2}^{\prime \prime}(u)$ such that $v \leq x \leq r(x) \leq p$ and $w \leq y \leq r(y) \leq q$, all inequalities in the $C^{*}$-algebra $Z_{2}^{\prime \prime}(u)$. We leave the rest as an exercise.

One might ask if there is an Urysohn lemma for the case that one of the tripotents is merely "closed", as in [4, Proposition 2.6]. It is clear by the methods above that this equivalent to asking if every closed tripotent is dominated by an open one, and we are not sure if the latter holds in all TROs.

We end this section with a couple of results which are interesting in their own right, and which we will need later.

Lemma 3.21. Let $A$ be a $C^{*}$-algebra and $x=\left[x_{i j}\right] \in M_{n}(A)_{+}$. Then

$$
\left(\bigwedge_{i} u\left(x_{i i}\right)\right) \otimes I_{n} \leq u(x) \leq x \leq r(x) \leq\left(\bigvee_{i} r\left(x_{i i}\right)\right) \otimes I_{n} .
$$

Proof. By an obvious induction argument it suffices to prove the case that $n=2$. Let $p=\bigvee_{i} r\left(x_{i i}\right)$. Clearly $x_{i i} p=x_{i i}$ for each $i$. We claim that $x_{i j} p=x_{i j}$ for each $i, j$. Note $p^{\perp} r\left(x_{22}\right) p^{\perp}=0$. Thus $0 \leq\left(1 \oplus p^{\perp}\right) x\left(1 \oplus p^{\perp}\right)$, which forces, by elementary operator theory, that $x_{12} p^{\perp}=0$. A similar argument shows that $x_{21} p^{\perp}=0$. Thus $x\left(p \otimes I_{n}\right)=x$, so that $r(x) \leq p \otimes I_{n}$ as desired.

By elementary operator theory, if $q$ is a projection with $q x q=q$ then $q x(1-q)=(1-q) x q=0$. Using the principle in the first paragraph of the proof again, and the fact that $u\left(x_{i i}\right)=x_{i i} u\left(x_{i i}\right)=u\left(x_{i i}\right) x_{i i}$, we find that $x_{i j} u\left(x_{i i}\right)=0$ if $i \neq j$. This gives $x\left(\left(\bigwedge_{i} u\left(x_{i i}\right)\right) \otimes I_{n}\right)=\left(\bigwedge_{i} u\left(x_{i i}\right)\right) \otimes I_{n}$, so that $\left(\bigwedge_{i} u\left(x_{i i}\right)\right) \otimes I_{n} \leq u(x)$.

In the following result, for a $*$-TRO $Z$ we write $\widetilde{\mathcal{L}}$ for the $C^{*}$-subalgebra of $L(Z)$ whose two main diagonal entries are equal, and whose off-diagonal entries are equal (see e.g. [13, Section 2]). We call this the restricted linking algebra.

Lemma 3.22. Let $Z$ be $a *-T R O$, and let $p$ be a selfadjoint projection in the center of $\widetilde{\mathcal{L}^{\prime \prime}}$. Then $p$ is of the form $\frac{1}{2}\left(I(u)+I(u)^{2}\right) \oplus^{\infty}\left(q \otimes e_{11}+q \otimes e_{22}\right)$, 
where $u$ is a central selfadjoint tripotent in $Z^{\prime \prime}$, and $q$ is a central projection in $\left(Z^{2}\right)^{\prime \prime}$ such that $q u=u q=0$. If $p$ is open in $\widetilde{\mathcal{L}}^{\prime \prime}$ then $q$ is open in $\left(Z^{2}\right)^{\prime \prime}$.

Proof. Let $\Theta$ as usual be the canonical $*$-automorphism on $L(Z)$, namely changing the sign of the off-diagonal corners. Then $\Theta(p)$ is central too. Let $u=p-\Theta(p)$. A computation shows that $u$ is a tripotent, and $\frac{1}{2}\left(u+u^{2}\right)=$ $p-p \Theta(p)$. Since $p$ is the orthogonal sum of $p \Theta(p)$ and $\frac{1}{2}\left(u+u^{2}\right)$, it is easy to see the first assertion. If $p$ is open then so is $\Theta(p)$ by an obvious argument using the canonical $*$-automorphism on $L(Z)$. Since a product of central open projections is open, $p \Theta(p)=q \otimes e_{11}+q \otimes e_{22}$ is open in $\widetilde{\mathcal{L}^{\prime \prime}}$, and now it is easy to see that $q$ is open in $\left(Z^{2}\right)^{\prime \prime}$.

REMARK. Simple examples show that in the last lemma one cannot hope that $u$ is an open tripotent necessarily, if $p$ is open, even if $Z$ is the commutative $C^{*}$-algebra $C([0,1])$. See, however, Lemma 4.7 for something along this line.

4. Maximal cones on TROs. We will need to develop TRO generalizations of facts from [13, Section 5]. The reader may wish to follow along with that paper.

If $Z$ is a TRO, and if $u$ is a tripotent in $Z$, then $u \otimes I_{n}$ is a tripotent in $M_{n}(Z)$, and hence there is an associated $C^{*}$-algebra $M_{n}(Z)_{2}\left(u \otimes I_{n}\right)$, which equips $M_{n}(Z)$ with a cone $\mathfrak{c}_{n}$. Of course $\mathfrak{c}_{1}=\mathfrak{c}_{u}$. Indeed,

$$
\mathfrak{c}_{n}=\mathfrak{c}_{u \otimes I_{n}}=\left\{\left[x_{i j}\right] \in M_{n}\left(Z_{2}(u)\right):\left[u^{*} x_{i j}\right] \geq 0\right\}=M_{n}\left(Z_{2}(u)\right)_{+} .
$$

Similarly, if $u$ is a tripotent in $Z^{\prime \prime}$ then we have a canonical natural cone on $M_{n}(Z)$ :

$$
\mathfrak{d}_{n}=\mathfrak{d}_{u \otimes I_{n}}=\left\{\left[x_{i j}\right] \in M_{n}(Z(u)):\left[u^{*} x_{i j}\right] \geq 0\right\}=M_{n}(Z(u))_{+} .
$$

Sometimes we will write $\mathfrak{d}_{u}$ for the entire sequence $\left(\mathfrak{d}_{u \otimes I_{n}}\right)$, and similarly for $\mathfrak{c}_{u}$.

We will need a fact about quotients of TROs. First recall that if $Z$ is a TRO, and if $J$ is a ternary ideal in $Z$, then $Z / J$ may again be viewed as a TRO (see e.g. [12, Section 8.3]).

LEMma 4.1. If $J$ is a ternary ideal in a naturally ordered TRO $Z$, then the TRO Z/J possesses a natural cone for which the canonical quotient ternary morphism $Z \rightarrow Z / J$ is completely positive.

Proof. If $Z$ is a TRO in a $C^{*}$-algebra $A$, we consider $Z^{\prime \prime}$ as a TRO in the $W^{*}$-algebra $A^{\prime \prime}$. Now $J^{\perp \perp}$ is a weak* closed ternary ideal in $Z^{\prime \prime}$, and hence equals $Z^{\prime \prime} q$ for a central projection $q$ in $\left(Z^{*} Z\right)^{\prime \prime}$, as is well known (for example, it is a special case of [14, Theorem 7.4(vi)]). If $p=1-q$ then $(Z / J)^{\prime \prime} \cong Z^{\prime \prime} / J^{\perp \perp} \cong Z^{\prime \prime} p$. We may thus identify $Z / J$ as a TRO inside the WTRO $Z^{\prime \prime} p$. This endows $Z / J$ with natural matrix cones. Let $q_{J}: Z \rightarrow Z / J$ 
be the quotient ternary morphism. If $z \in Z_{+}$then $z \geq 0$ in $A^{\prime \prime}$, and so $z=\left(z^{*} z\right)^{1 / 2}$. Thus $z p=\left(z^{*} z\right)^{1 / 2} p=p\left(z^{*} z\right)^{1 / 2} p \geq 0$, and so $q_{J}(z)$ is in the cone just defined in $Z / J$. A similar argument applies to matrices, so that $q_{J}$ is completely positive.

LEMMA 4.2. Let $Z$ be a TRO with matrix cones $\mathfrak{C}=\left(\mathfrak{C}_{n}\right)$ for which there exists a completely positive complete isometry from $Z$ into a $C^{*}$-algebra. Then the given cones $\mathfrak{C}$ in $Z$ are contained in a natural cone for $Z$.

Proof. Just as in [13, Lemma 5.3].

Definition 4.3. We say that an operator space ordering $\left(\mathfrak{c}_{n}\right)$ on an operator space $X$ is maximal, or that $X$ is maximally ordered, if $\left(\mathfrak{c}_{n}\right)$ is maximal amongst the operator space orderings on $X$. This is equivalent to saying that every completely positive complete isometry $X \rightarrow B$ into a $C^{*}$-algebra is a complete order embedding.

It follows from Lemma 4.2 that the maximal (operator space) orderings on a TRO are precisely the maximal natural orderings.

THEOREM 4.4. Suppose $Z$ is a TRO with an operator space ordering. Then $Z$ has a maximal (operator space) ordering majorizing the given one, and this cone is natural.

Proof. Just as in [13, Theorem 5.4], but including an appeal to Proposition 2.12 .

As mentioned after Corollary 2.8, natural dual cones in a WTRO $W$ correspond bijectively to tripotents in $W$. This gives a very satisfactory characterization of the maximal natural dual cones. Maximal natural dual cones in a WTRO $W$ correspond to maximal tripotents, which are exactly the extreme points of $\operatorname{Ball}(W)$. Indeed, the extreme points of $\operatorname{Ball}(W)$ are well known to be the tripotents such that $\left(1-u u^{*}\right) W\left(1-u^{*} u\right)=(0)$. Any such tripotent is maximal, since if $v \geq u$ then

$$
0=\left(1-u u^{*}\right) v\left(1-u^{*} u\right)=(v-u)\left(1-u^{*} u\right)=v-u .
$$

Conversely, if the WTRO $\left(1-u u^{*}\right) W\left(1-u^{*} u\right)$ is not $(0)$ then it has a nonzero tripotent $w \perp u$, and $w+u \geq u$. Thus $u$ is not maximal.

In a TRO $Z$, maximal natural cones correspond to maximal open tripotents. These exist by Zorn's lemma, since any increasing chain of open tripotents is bounded above by an open tripotent (Proposition 2.12). We consider maximal open tripotents in Theorem 4.5 below, also settling an issue raised in [13, Section 5], which we now describe.

Let $A$ be a $C^{*}$-algebra and let $p$ and $q$ be an open and a closed central projection in $A^{\prime \prime}$. We say that $q$ is contained in the boundary of $p$ if $p \perp q$ and 
if whenever $r$ is an open central $\left({ }^{2}\right)$ projection in $A^{\prime \prime}$ which is perpendicular to $p$, then $r$ is perpendicular to $q$. We shall not use this, but if $q+p$ is closed, which will be the case for us below, then it is easy to see that $q$ is contained in the boundary of $p$ iff $q+p$ is the smallest closed central projection dominating $p$. In [13, Proposition 5.11] it was shown that if $Z$ is a $*-$ TRO and $u$ is a selfadjoint central open tripotent in $Z^{\prime \prime}$, then $u$ is maximal amongst the selfadjoint central open tripotents in $Z^{\prime \prime}$ if $1-u^{2}$ is contained in the boundary $\left(^{3}\right)$ of both $\frac{1}{2}\left(u+u^{2}\right)$ and $\frac{1}{2}\left(-u+u^{2}\right)$. Here the $C^{*}$-algebra $A$ is $Z+Z^{2}$. It was also noted there that the converse of this is true in the "commutative case"; however, an inspection of the proof of this converse (see [13, Corollary 6.8]) shows that we were also assuming there that $Z \cap Z^{2}=(0)$. This is not a serious restriction, since any $*$ TRO is ternary $*$-isomorphic to one satisfying this property, and below we shall always assume that $Z \cap Z^{2}=(0)$ when we use the phrase " $1-u^{2}$ is contained in the boundary of $\frac{1}{2}\left(u+u^{2}\right)$ ". It was suggested in [13] that such a "contained in the boundary" condition might characterize maximal selfadjoint central open tripotents for any $*$-TRO. By results in that paper, such a characterization would immediately give a characterization of the maximal ordered operator space cones, and thus also the maximal cones which are natural in the sense of [13], on any $*$-TRO.

To motivate the value of having such a characterization, it is very instructive to look at a commutative example studied in [13, Section 6]. Let $S^{2}$ be the unit sphere, and $Z$ the $*$-TRO $\left\{f \in C\left(S^{2}\right): f(-x)=-f(x)\right\}$. In this case open selfadjoint tripotents $u$ in $Z^{\prime \prime}$ correspond precisely to open subsets $U$ of the sphere (called blue), which do not intersect $-U$ (called red). Suppose that $S^{2} \backslash(U \cup(-U))$ is colored black. The "contained in the boundary" characterization discussed in the last paragraph says precisely $\left({ }^{4}\right)$ that $u$ (and hence the associated ordering of $Z$ ) is maximal iff the black region is the boundary of the red region (and hence also of the blue region). Thus, for example, a sphere whose top hemisphere is red and whose bottom hemisphere is blue, with a black equator line, is maximal; whereas if one were to thicken the equator to a black band one loses maximality. From the geometry of such examples, it seems clear that one could not improve on this characterization.

$\left({ }^{2}\right)$ Dealing with central projections here yields a simpler characterization without introducing any additional complications.

$\left({ }^{3}\right)$ We shall not use this, but it is easy to see that $1-u^{2}$ is contained in the boundary of $\widehat{u}=\frac{1}{2}\left(u+u^{2}\right)$ iff $\frac{1}{2}\left(u^{2}-u\right)^{\perp}$ is the smallest closed central projection dominating $\frac{1}{2}\left(u+u^{2}\right)$.

$\left({ }^{4}\right)$ This is misstated in the fourth last line of p. 709 of [13], but the typographical error should be clear in the context. 
In the "noncommutative case" it is unfortunately not true that if $u$ is maximal amongst the selfadjoint central open tripotents in $Z^{\prime \prime}$ then $1-u^{2}$ is contained in the boundary of $\frac{1}{2}\left(u+u^{2}\right)$. For example, take $Z$ to be the subspace of $M_{2}$ with main diagonal entries zero. In this case, $u=0$ is a maximal selfadjoint central open tripotent in $Z^{\prime \prime}$, but $r=I_{2}$ satisfies $r \frac{1}{2}\left(u+u^{2}\right)=0$ but $r\left(1-u^{2}\right)=r \neq 0$. It does not help if we replace $A=Z+Z^{2}$ by the restricted linking $C^{*}$-algebra $\widetilde{\mathcal{L}}$ mentioned at the end of Section 4 ; indeed, this situation is equivalent since $Z+Z^{2}$ is $*$-isomorphic to $\widetilde{\mathcal{L}}$ if $Z \cap Z^{2}=(0)$.

This problem can be remedied in several ways. For example, we can put a restriction on the projections $r$ considered in the definition of "contained in the boundary". If $A=Z+Z^{2}$ and $Z \cap Z^{2}=0$, we say that a projection $r$ in $A^{\prime \prime}$ is antisymmetric if $\Theta^{\prime \prime}(r) \perp r$. Here $\Theta: A \rightarrow A$ is the period 2 *-automorphism $\Theta(z+a)=a-z$ for $z \in Z$ and $a \in Z^{2}$. If $r$ is a projection (resp. central projection) in $A^{\prime \prime}$, then it is easy to see that $r$ is antisymmetric iff $r=\frac{1}{2}\left(v+v^{2}\right)$ for a selfadjoint tripotent (resp. central selfadjoint tripotent) $v \in Z^{\prime \prime}$. If $r$ is an open central projection in $A^{\prime \prime}$, then it is easy to check using Lemma 3.22 and [13, Proposition 4.18] that $r$ is antisymmetric iff $r$ dominates no nontrivial open central projection in $\left(Z^{2}\right)^{\prime \prime}$. We say that $q$ is antisymmetrically contained in the boundary of $p$ if $p \perp q$ and whenever $r$ is an open antisymmetric central projection in $A^{\prime \prime}$ which is perpendicular to $p$, then $r \perp q$.

For a general TRO $Z$, we use the definition of antisymmetric projections from the introduction. Let $A=L(Z)$, and let $p$ and $q$ be respectively open and closed projections in $A^{\prime \prime}$. We say that $q$ is antisymmetrically contained in the boundary of $p$ if $p \perp q$ and whenever $r$ is an open antisymmetric projection in $A^{\prime \prime}$ which is perpendicular to $p$, then $r \perp q$.

The following is a characterization of maximal open tripotents in a TRO:

TheOREM 4.5. Let $Z$ be a TRO. Suppose $u$ is an open tripotent in $Z^{\prime \prime}$. Then $u$ is maximal amongst the open tripotents if and only if $1-I(u)^{2}$ is antisymmetrically contained in the boundary of $\widehat{u}$.

Proof. Since $u$ is maximal iff $-u$ is maximal, we may replace $\widehat{u}$ by $\breve{u}$. Suppose that $1-I(u)^{2}$ is antisymmetrically contained in the boundary of $\breve{u}$. If $v \geq u$ and if $v$ is open, then $v$ commutes with $u$, and $r=\widehat{v}$ is (by Theorem 2.10) an open projection in $L(Z)^{\prime \prime}$. It is easy to check that $r \breve{u}=0$. Thus $r\left(1-I(u)^{2}\right)=0$, which gives $v=v u^{*} u=u$. Thus $u$ is maximal.

Conversely, suppose that $u$ is a maximal open tripotent in $Z^{\prime \prime}$. Suppose that $r=\widehat{v}$ is an open antisymmetric projection with $r \breve{u}=0$. From the commutator relations coming from this last equality, we see that $u \vee v$ exists by Lemma 3.7, and thus it is open by Proposition 3.8. Since $u$ is maximal, 
$u=u \vee v$, and so $v \leq u$. Hence $v=v u^{*} u$. Inspection now reveals that $\widehat{v}\left(1-I(u)^{2}\right)=0$.

The following result, whose proof we omit since it is essentially the same as the proof of Theorem 4.5, characterizes maximal cones in a $*$-TRO.

Proposition 4.6. Let $Z$ be $a *-T R O$, and let $u$ be a selfadjoint central open tripotent in $Z^{\prime \prime}$. Then $u$ is maximal amongst the selfadjoint central open tripotents if and only if $1-u^{2}$ is antisymmetrically contained in the boundary of $\frac{1}{2}\left(u+u^{*}\right)$.

REMARK. It follows easily from what we have done that the conjecture from [13] that we have been discussing is true for the class of TROs $Z$ which have the following property: whenever $p$ and $q$ are respectively open and closed central projections in $\left(Z+Z^{2}\right)^{\prime \prime}$, which are not orthogonal to each other, then $p$ dominates an antisymmetric open central projection $r$ which is not orthogonal to $q$. This condition is always satisfied in the "commutative case" of $[13$, Section 6$]$. We remark too that the fact that the conjecture is true in this commutative case, also follows from the result below (since in the commutative case, in the notation below, $r$ is necessarily antisymmetric, for if it were not then the existence of $v$ below contradicts the maximality of $u$ ).

Lemma 4.7. Let $Z$ be $a *-T R O$ with $Z \cap Z^{2}=(0)$. Suppose that $r$ is an open central projection in $\left(Z+Z^{2}\right)^{\prime \prime}$ which is perpendicular to $\widehat{u}=\frac{1}{2}\left(u+u^{2}\right)$ for a maximal central selfadjoint open tripotent $u \in Z^{\prime \prime}$. Then either $r$ is antisymmetric (and thus orthogonal to $1-u^{2}$ ), or there exists a nonzero selfadjoint open tripotent $v \in Z^{\prime \prime}$ which is perpendicular to $u$ and $r \geq \widehat{v}$.

Proof. By Lemma 3.22, $r=q+\frac{1}{2}\left(u+u^{2}\right)$ where $q$ is an open central projection in $Z^{2}, u$ is a central selfadjoint tripotent, and $q \perp u$. If $q=0$ then we are done: $r$ is antisymmetric and is orthogonal to $1-u^{2}$ by Proposition 4.6. If not, suppose that $x_{\lambda}$ is a net in $Z^{2}$ converging up to $q$. Clearly there exists an element $y \in Z$, which we can take to be selfadjoint, and a $\lambda$ with $z=x_{\lambda} y x_{\lambda} \neq 0$ (for otherwise, taking a strong limit in $\left(Z+Z^{2}\right)^{\prime \prime}$, we have $q y q=q y=0$ for all $y \in Z$, so that $q=0)$. Of course $r(z)$ is open in $Z^{\prime \prime}$. We have $z=z q \perp u$, and so $r(z) \perp u$ and $r(z) q=r(z)$. Clearly $\widehat{r(z)} \leq q \leq r$.

Let $Z$ be a $*$-TRO. For any set $S \subset Z^{\prime \prime}$, we denote by $S_{\vdash}$ the set $\{x \in Z$ : $y x=x y=0 \forall y \in S\}$. If $\mathfrak{c}$ is a natural dual cone in $Z^{\prime \prime}$ in the sense of [13], then $\mathfrak{c}=\mathfrak{c}_{u}$ for an open central selfadjoint tripotent $u \in Z^{\prime \prime}$. In this case, $\mathfrak{c}_{\vdash}=u_{\vdash}$, and this is a ternary $*$-ideal in $Z$. It follows from [13, Lemma 3.4] that $u_{\vdash}=\{z \in Z: r z=z\}$, and $\left(u_{\vdash}\right)^{\perp \perp}=r Z^{\prime \prime}$, for an open central projection in $\left(Z^{2}\right)^{\prime \prime}$ such that $r z=z p$ for all $z \in Z$. We claim that $r u=0$. Indeed, $r u \in r Z^{\prime \prime}=\left(u_{\vdash}\right)^{\perp \perp}$, and if $x_{t} \in u_{\vdash}$ with $x_{t} \rightarrow r u$ weak ${ }^{*}$, then $0=u^{2} x_{t} \rightarrow r u$, so that $r u=0$. 
COROLlary 4.8. If $Z$ is a $*$-TRO, and $u$ is an open selfadjoint tripotent in $Z^{\prime \prime}$ then $1-u^{2}$ is contained in the boundary of $\frac{1}{2}\left(u+u^{2}\right)$ iff $u$ is maximal amongst the open selfadjoint central tripotents and $u_{\vdash}=(0)$.

Proof. $(\Rightarrow)$ Under this hypothesis, $u$ is maximal as before. If $u_{\vdash} \neq 0$, then if $r$ is as above Corollary 4.8 then $r \neq 0$. On the other hand, $r \frac{1}{2}\left(u+u^{2}\right)$ $=0$, and so $r\left(1-u^{2}\right)=r=0$, a contradiction.

$(\Leftarrow)$ If $v$ is open and perpendicular to $u$, and if $x_{t} \rightarrow v$ weak* with $x_{t} \in Z(v)$, then $x_{t} \in u_{\vdash}=(0)$. Thus $v=0$. The result then follows from Lemma 4.7.

We now isolate the class of $*$-TROs for which the conjecture from [13] is correct.

Definition 4.9. A $*$-TRO $Z$ is said to be completely orderable if for every natural dual cone $\mathfrak{c}$ in $Z^{\prime \prime}$, either $\mathfrak{c}_{\vdash}=(0)$ or $\mathfrak{c}_{\vdash}$ has a nontrivial natural ordering in the sense of [13].

LEMMa 4.10. A*-TRO $Z$ is completely orderable iff for every maximal open selfadjoint central tripotent $u \in Z^{\prime \prime}$ we have $u_{\vdash}=(0)$.

Proof. We may assume without loss of generality that $Z \cap Z^{2}=(0)$, and we denote $Z+Z^{2}$ by $A$.

Suppose that $u_{\vdash}=(0)$ for every maximal open selfadjoint central tripotent $u$. If $Z$ is not completely orderable, then there is an open central selfadjoint tripotent $u$ such that $u_{\vdash}$ is not orderable (and nontrivial). Write $u_{\vdash}=\{z \in Z: r z=z\}$ as above Corollary 4.8. Let $v$ be a maximal central open tripotent with $v \geq u$. Since by hypothesis $v_{\vdash}=(0)$, we must have $v r \neq 0$ (for if $v r=0$ then if $0 \neq x \in u_{\vdash}$ then $x v=x r v=0=v r x=v x$, so that $\left.0 \neq x \in v_{\vdash}=(0)\right)$. Let $w=v r$, a central selfadjoint tripotent in $Z^{\prime \prime}$ which is perpendicular to $u$ by the line above Corollary 4.8. Note that $r$ is also an open central projection in $A^{\prime \prime}$, and that $v$ is an open selfadjoint central tripotent in $A^{\prime \prime}$, so that $w$ is an open selfadjoint central tripotent in $A^{\prime \prime}$ by e.g. Corollary 3.9. Suppose that $\left(x_{\lambda}+y_{\lambda}\right)$ is an increasing net in $A(w)_{+}$which converges to $w$, with $x_{\lambda} \in Z^{2}$ and $y_{\lambda} \in Z$. Let $\Theta$ be the map mentioned above Theorem 4.5. Since $-\Theta$ is a ternary isomorphism on $A$ and $-\Theta(w)=w$, we have $-\theta\left(A(w)_{+}\right)=A(w)_{+}$by Proposition 3.5. Hence, $-\Theta\left(x_{\lambda}+y_{\lambda}\right)=-x_{\lambda}+y_{\lambda}$ is also an increasing net with limit $w$ in $A(w)_{+}$. Thus $y_{\lambda} \in A(w)_{+} \subset A_{2}^{\prime \prime}(w)_{+}$. Since $y_{\lambda} \in Z_{2}^{\prime \prime}(w)$, and since $Z_{2}^{\prime \prime}(w)$ is a $C^{*}$-subalgebra of $A_{2}^{\prime \prime}(w)$, we see that $y_{\lambda} \in Z_{2}^{\prime \prime}(w)_{+} \cap Z=\mathfrak{d}_{w}$. Since $y_{\lambda} \rightarrow w$ weak* $^{*}$ we conclude that $w$ is open in $Z^{\prime \prime}$. Since $w$ is perpendicular to $u$ we must have $Z(w) \subset u_{\vdash}$, and so $w$ is a tripotent in $\left(u_{\vdash}\right)^{\perp \perp}$ which is open in that space. By [13, Corollary 5.7] we see that $u_{\vdash}$ is orderable. This is a contradiction, and so $Z$ is completely orderable. 
For the other direction, suppose that $Z$ is completely orderable. If $u$ is a maximal open selfadjoint central tripotent in $Z^{\prime \prime}$ with $u_{\vdash} \neq(0)$, then $u_{\vdash}$ has a nontrivial natural ordering. Thus by [13], there is a nontrivial open selfadjoint central tripotent $w \in\left(u_{\vdash}\right)^{\prime \prime} \cong\left(u_{\vdash}\right)^{\perp \perp}$. This tripotent $w$ is also an open selfadjoint tripotent in $Z^{\prime \prime}$. Since $\left(u_{\vdash}\right)^{\perp \perp}=r Z^{\prime \prime}$ as above Corollary 4.8, and since $w r^{\perp}=0$, it is easy to see that $w$ is central in $Z^{\prime \prime}$. Since $w \in\left(u_{\vdash}\right)^{\perp \perp}$, we have $w \perp u$. Since $u+w \geq u$ we have arrived at a contradiction. Thus $u_{\vdash}=(0)$.

THEOREM 4.11. If $Z$ is a completely orderable $*$-TRO with $Z \cap Z^{2}=(0)$, then an open selfadjoint central tripotent $u \in Z^{\prime \prime}$ is maximal amongst the open selfadjoint central tripotents iff $1-u^{2}$ is contained in the boundary of $\frac{1}{2}\left(u+u^{2}\right)$. If $Z$ is not completely orderable, then there exists a maximal open selfadjoint central tripotent $u$ such that $1-u^{2}$ is not contained in the boundary of $\frac{1}{2}\left(u+u^{2}\right)$.

Proof. If $Z$ is completely orderable, and if $u$ is a maximal open selfadjoint central tripotent in $Z^{\prime \prime}$, then $u_{\vdash}=(0)$, and so $1-u^{2}$ is contained in the boundary of $\frac{1}{2}\left(u+u^{2}\right)$ by Corollary 4.8 . The converse direction also follows from Corollary 4.8 .

Suppose that $Z$ is not completely orderable. By Lemma 4.10, there is a maximal open selfadjoint central tripotent $u$, with $u_{\vdash} \neq(0)$. By the lines above Corollary 4.8, there is a nonzero open central projection $r \in\left(Z^{2}\right)^{\prime \prime}$ with $r \perp u$. Thus $r$ is an open central projection in $A^{\prime \prime}$, and $r \perp \frac{1}{2}\left(u+u^{2}\right)$ but $r\left(1-u^{2}\right)=r \neq 0$.

REMARK. Commutative $*$-TROs are completely orderable, by results in $[13$, Section 6$]$ or by a simple direct argument. $C^{*}$-algebras also satisfy this condition. In this case, for any maximal open selfadjoint central tripotent $u$, we deduce that $u_{\vdash}$ is a two-sided ideal. If $p$ is the support projection of this ideal, then $p \perp u$. Thus $u+p$ is an open selfadjoint central tripotent dominating $u$. By maximality of $u$ we have $p=0$ and $u_{\vdash}=(0)$.

5. Cones on operator spaces and the Shilov boundary. In this section we study an operator space $X$ with a given cone $\mathfrak{c}$, or with a sequence of matrix cones $\mathfrak{c}_{n} \subset M_{n}(X)$. One of the advantages of our approach is that it can be done in either of these two settings, that is, for nonmatricial or for matricial cones. Nonetheless we will usually focus on the matricial cone case, leaving the nonmatricial case to the reader, with the following lemma being an exception:

Proposition 5.1. Suppose that $X$ is an operator space with a cone $\mathfrak{c}$ which densely spans $X$, and that $i: X \rightarrow B$ is a positive complete isometry from $X$ into a $C^{*}$-algebra. Then the TRO $W$ generated by $i(X)$ is a $C^{*}$ - 
subalgebra of $B$, and $W^{\prime \prime}$ is a $W^{*}$-subalgebra of $B^{\prime \prime}$. Moreover, if $u$ is the tripotent associated with the natural cone of $W$, then $W=W(u)$.

Proof. If $x \in \mathfrak{c}$ then $i(x) \in W \cap B_{+} \subset J(W)$ (see Lemma 2.1). Hence $i(X) \subset J(W)$, so that $W \subset J(W)$. Thus $W=J(W)$ is a $C^{*}$-subalgebra of $B$. The other assertions are obvious.

LEMMA 5.2. Let $\left(\mathfrak{c}_{n}\right)$ be an operator space ordering on an operator space $X$, and let $i: X \rightarrow B$ be a completely positive complete isometry into a $C^{*}$-algebra. If $W=\langle i(X)\rangle$, set $u=\bigvee_{x \in \mathfrak{c}_{1}} r(i(x))$, an open tripotent in $W^{\prime \prime}$. Then $i_{n}\left(\mathfrak{c}_{n}\right) \subset \mathfrak{d}_{u \otimes I_{n}}$.

Proof. We need to show that if $\left[x_{i j}\right] \in \mathfrak{c}_{n}$, and $x=\left[i\left(x_{i j}\right)\right]$, then $x \in$ $\mathfrak{d}_{u \otimes I_{n}}$. By Lemma 3.1 this is equivalent to saying that $r(x) \leq u \otimes I_{n}$, which in turn follows from Lemma 3.21.

As we said early in Section 4 , the cone $\mathfrak{d}_{u \otimes I_{n}}$ in the last lemma is the natural cone in $M_{n}(Z)$ corresponding to the tripotent $u$ (that is, it is the $n$th cone in the sequence of matrix cones associated with $u$ ).

Next, we construct an ordered version of the "noncommutative Shilov boundary" or "ternary envelope" [26, 12]. We recall its universal property, which we use frequently. The ternary envelope of an operator space $X$ is a pair $(\mathcal{T}(X), j)$ consisting of a TRO $\mathcal{T}(X)$ and a completely isometric linear map $j: X \rightarrow \mathcal{T}(X)$ such that $\mathcal{T}(X)$ is generated by $j(X)$ as a TRO (that is, there is no closed subTRO containing $j(X)$ ), and which has the following property: given any completely isometric linear map $i$ from $X$ into a TRO $Z$ which is generated by $i(X)$, there exists a (necessarily unique and surjective) ternary morphism $\theta: Z \rightarrow \mathcal{T}(X)$ such that $\theta \circ i=j$. A pair $(\mathcal{T}(X), j)$ with this universal property is unique up to ternary isomorphism "fixing the copy of $X$ ". By considering simple examples (for example, orderings on $\mathbb{C} !$ ), one quickly sees that if one wants an ordered version of this that works for operator spaces with sensible positive cones, the embeddings $i: X \rightarrow Z$ occurring in the universal property above cannot be allowed to be arbitrary completely positive complete isometries, or even arbitrary completely isometric complete order embeddings (unless we have a strong extra condition, such as $X_{+}$densely spanning $\left.X\right)$. We will usually need to limit the size of the cone of $Z$.

More specifically, suppose that $X$ is an operator space possessing a cone $\mathfrak{c}$ (resp. sequence of matrix cones $\mathfrak{c}=\left(\mathfrak{c}_{n}\right)$ ) such that there is a positive (resp. completely positive) complete isometry $i: X \rightarrow B$ into a $C^{*}$-algebra $B$. Then we can assign a canonical cone (resp. sequence of matrix cones) to the ternary envelope $(\mathcal{T}(X), j)$, namely the intersection of all natural cones containing $j(\mathfrak{c})$ (resp. $\left.\left(j\left(\mathfrak{c}_{n}\right)\right)\right)$. We call $\mathcal{T}(X)$ equipped with this cone structure the ordered ternary envelope $\mathcal{T}^{\mathrm{O}}(X)$. To see that there exists at least 
one such cone, note that if $i: X \rightarrow B$ is as above, and if $W$ is the TRO generated by $i(X)$, then by the universal property of the ternary envelope above, there is a ternary morphism $\theta: W \rightarrow \mathcal{T}(X)$ with $\theta \circ i=j$. Thus $\mathcal{T}(X)$ is ternary isomorphic to a quotient of $W$. By Lemma 4.1, this quotient of $W$ has a natural cone containing the image of $i(\mathfrak{c})$ in the quotient. Hence $\mathcal{T}(X)$ has a natural cone containing $j(\mathfrak{c})$ (resp. containing the sequence $\left.\left(j_{n}\left(\mathfrak{c}_{n}\right)\right)\right)$. In particular, $j: X \rightarrow \mathcal{T}^{\circ}(X)$ is positive (resp. completely positive). It is easy to see (using Lemma 5.2 in the matricial cone case) that the open tripotent corresponding to the ordering which we have given $\mathcal{T}^{\circ}(X)$ is $u=\bigvee_{x \in \mathfrak{c}} r(j(x))$.

If $\left(\mathcal{T}^{\circ}(X), j\right)$ is the ordered ternary envelope of $(X, \mathfrak{c})$, then we define the order completion of $\mathfrak{c}$ to be the cone $\overline{\mathfrak{c}}=j^{-1}\left(\mathcal{T}^{\circ}(X)_{+} \cap j(X)\right)$ in $X$. It is of interest to know when $\mathfrak{c}$ is complete, that is, $\mathfrak{c}=\overline{\mathfrak{c}}$, or equivalently, that the canonical embedding of $X$ in $\mathcal{T}^{\circ}(X)$ is a (complete) order embedding. Later in this section we will give some sufficient conditions for this.

The following theorem is stated in the matricial cone case; in the nonmatricial case delete the occurrences of the word "completely", and ignore matrix cones.

TheOREM 5.3. Suppose that $X$ is an operator space with matrix cones $\mathfrak{c}=\left(\mathfrak{c}_{n}\right)$, and that $i: X \rightarrow B$ is a completely positive complete isometry from $X$ into a $C^{*}$-algebra, such that if $W$ is the TRO generated by $i(X)$ then there is no smaller natural cone than $W \cap B_{+}$on $W$ which contains $i(\mathfrak{c})$ (or, equivalently, that $W \cap B_{+}$is the intersection of the natural cones containing $i(\mathfrak{c}))$. Let $\left(\mathcal{T}^{\circ}(X), j\right)$ be the ordered ternary envelope of $X$. Then there exists a completely positive ternary morphism $\theta: W \rightarrow \mathcal{T}^{\circ}(X)$ such that $\theta \circ i=j$. Moreover, $\theta$ restricts to a surjective $*$-homomorphism between the $C^{*}$-algebras associated with the natural orderings. In particular, $\theta\left(W \cap B_{+}\right)=\mathcal{T}^{\circ}(X)_{+}$.

Proof. Let $\mathfrak{c}$ be the cone on $X$. By the universal property of $\mathcal{T}(X)$, there exists such a map $\theta$; we need to prove that $\theta$ is completely positive. Note that all of the positivity in $Z$ resides in the $W^{*}$-algebras $M_{n}\left(Z_{2}^{\prime \prime}(u)\right)$, where $u$ is the open tripotent giving the ordering on $W$. Similarly for $\mathcal{T}(X)$, and write $w$ for the open tripotent giving its ordering. Let $\pi$ be $\theta^{\prime \prime}$ restricted to $Z_{2}^{\prime \prime}(u)$, which is a weak* continuous $*$-homomorphism (and therefore automatically completely positive) from $Z_{2}^{\prime \prime}(u)$ onto $Z_{2}^{\prime \prime}(\pi(u))$. Products below are taken in those algebras. It suffices to show that $\pi(u)=w$. Observe that $\pi\left(i(x)^{2 n-1}\right)=j(x)^{2 n-1}$ for any $x \in \mathfrak{c} \cap \operatorname{Ball}(X)$ and $n \in \mathbb{N}$. Using the fact stated after Lemma 3.3, we see that $\pi\left(i(x)^{1 /(2 n-1)}\right)=j(x)^{1 /(2 n-1)}$, and in the weak* limit, $\pi(r(i(x)))=r(j(x))$. Taking suprema, since weak* continuous $*$-homomorphisms preserve suprema, we know that $\pi(u)$ is the supremum in the $W^{*}$-algebra $Z_{2}^{\prime \prime}(\pi(u))$ of the projections $r(j(x))$ in that 
algebra. By definition of $w$, we have $w \leq \pi(u)$. Hence $w$ is a projection in $Z_{2}^{\prime \prime}(\pi(u))$, and now it is clear that $w=\pi(u)$. The desired surjectivity follows from Proposition 3.5.

Remark. The ordering we have given to $\mathcal{T}(X)$ does not depend on the particular ternary envelope chosen. This follows immediately for example from the universal property in the theorem.

The ordered noncommutative Shilov boundary is particularly nice in the case that $X$ has a densely spanning cone, for example this boundary is a $C^{*}$-algebra. In this case, it is easy to see that we may assume that $X$ is a selfadjoint operator space, and then the following result is in [11]. We include an alternative proof:

Corollary 5.4. Suppose that $X$ is an operator space with a cone $\mathfrak{c}$ which densely spans $X$, and that $i: X \rightarrow B$ is a positive complete isometry from $X$ into a $C^{*}$-algebra. Then the TRO A generated by $i(X)$ equals the $C^{*}$-subalgebra of $B$ generated by $i(X)$, and the hypothesis on the cone of $A$ in Theorem 5.3 holds automatically. Moreover, the ordered ternary envelope of $X$ is a $C^{*}$-algebra, and the canonical ternary morphism $\theta: A \rightarrow \mathcal{T}^{\circ}(X)$ such that $\theta \circ i=j$ is $a *$-homomorphism.

Proof. The first assertions follow from Proposition 5.1. Write $(D, j)$ for the ordered ternary envelope of $X$, viewed as a $C^{*}$-algebra. By the universal property of the ternary envelope, there exists a surjective ternary morphism $\theta: A \rightarrow D$ with $\theta \circ i=j$. Let $\mathfrak{d}$ be the intersection of the natural cones containing $i(\mathfrak{c})$. This is a natural cone in $A$, and its span is an inner ideal $J$ of $A$. Since $J$ is a subTRO too, $J$ contains the subTRO generated by $i(\mathfrak{c})$. Since $\mathfrak{c}$ densely spans $X, J$ contains the subTRO generated by $i(X)$. So $J$ $=A$, and it follows that $\mathfrak{d}=A_{+}$. Hence, and by the nonmatricial case of Theorem 5.3, $\theta$ is positive on $A$. By Lemma 1.1, $\theta$ is a $*$-homomorphism.

TheOREM 5.5. Suppose that $X$ is an operator space with matrix cones $\mathfrak{c}=\left(\mathfrak{c}_{n}\right)$, and let $j: X \rightarrow \mathcal{T}(X)$ be the canonical Shilov boundary embedding. Then there exists a completely positive complete isometry from $X$ into a $C^{*}$ algebra if and only if $\mathfrak{c} \subset j^{-1}\left(\mathfrak{d}_{u}\right)$, where $u$ is an open tripotent in $\mathcal{T}(X)^{\prime \prime}$.

If these hold and if $\mathfrak{c}_{1}$ densely spans $X$, then $\mathfrak{c}=j^{-1}\left(\mathfrak{d}_{u}\right)$ for some open tripotent $u$ in $\mathcal{T}(X)^{\prime \prime}$ if and only if $(X, \mathfrak{c})$ is maximally ordered.

Proof. The $(\Leftarrow)$ directions are easy, by looking at $j: X \rightarrow \mathcal{T}(X)$; equipping the latter space in the proof of the first "iff" with the natural cone $\mathfrak{d}_{u}$, and in the second "iff" with the natural cone of $\mathcal{T}^{\circ}(X)$.

For the $(\Rightarrow)$ direction of the first "iff", let $i: X \rightarrow B$ be a completely positive complete isometry from $X$ into a $C^{*}$-algebra $B$, and let $W=\langle i(X)\rangle$, the TRO generated by $i(X)$. Endow $W$ with the smallest natural cone which contains $i(\mathfrak{c})$. Let $\left(\mathcal{T}^{\circ}(X), j\right)$ be the ordered ternary envelope of $X$, and let 
$u$ be the open tripotent discussed above the last theorem. By that result, there exists a completely positive ternary morphism $\theta: W \rightarrow \mathcal{T}^{\circ}(X)$ such that $\theta \circ i=j$. This implies that $j(\mathfrak{c}) \subset \mathfrak{d}_{u}$.

Finally, if $\mathfrak{c}=j^{-1}\left(\mathfrak{d}_{u}\right)$ for some open tripotent, then this holds with $u$ the open tripotent corresponding to the natural cone of $\mathcal{T}^{\circ}(X)$. That is, $\mathfrak{c}=\overline{\mathfrak{c}}$. If $i(x) \geq 0$ for a completely positive complete isometry $i$ from $X$ into a $C^{*}$-algebra, then by Corollary 5.4 we have $j(x) \geq 0$, so that $x \in \overline{\mathfrak{c}}=\mathfrak{c}$. So $i$ is an order embedding, and similarly it is a complete order embedding.

REMARK. The nonmatricial cone case of the last result is valid with the same proof. Thus if $X$ is an operator space with a cone $\mathfrak{c}$, then there exists a positive complete isometry from $X$ into a $C^{*}$-algebra if and only if $\mathfrak{c} \subset \mathfrak{d}_{u}$, where $u$ is an open tripotent in $\mathcal{T}(X)^{\prime \prime}$.

The previous results have nice consequences concerning unitizations, which are explored a bit further in [11]. If $(X, \mathfrak{c})$ is an operator space with a densely spanning operator space cone, let $A=\mathcal{T}^{\circ}(X)$ be its ordered ternary envelope, which we now know is a $C^{*}$-algebra. Let $X^{1}$ be the span of $X$ and the identity of the $C^{*}$-algebra unitization of this $C^{*}$-algebra. Then if $H$ is a Hilbert space, and $i: X \rightarrow B(H)$ is a completely positive complete isometry, then it is easy to show, from the universal property of $\mathcal{T}^{\circ}(X)$, that there is a completely positive unital map from $i(X)+\mathbb{C} I_{H} \rightarrow X^{1}$ extending the canonical map $i(X) \rightarrow X$. One clearly has the following rigidity result: a unital completely positive linear map $\Phi: X^{1} \rightarrow B(H)$ is a complete order embedding if its restriction to $X$ is a completely isometric complete order embedding.

Corollary 5.6. Let $X$ be an operator space with an operator space cone which densely spans $X$. The following are equivalent:

(i) The embedding of $X$ in the unitization $X^{1}$ is a complete order embedding.

(ii) $X$ is maximally ordered.

(iii) The cone $\mathfrak{c}$ is complete (that is, the canonical embedding of $X$ in $\mathcal{T}^{\circ}(X)$ is a complete order embedding).

Proof. We already saw in Theorem 5.5 that (ii) $\Leftrightarrow($ iii), and the equivalence of (i) and (iii) is obvious from the definition of $X^{1}$.

For the next result we recall that a unital operator space is an operator space $X$ for which there exists a linear complete isometry $\varphi: X \rightarrow A$ into a unital $C^{*}$-algebra with $1_{A} \in \varphi(X)$. We will write 1 for $\varphi^{-1}\left(1_{A}\right)$. Any unital operator space has a $C^{*}$-envelope $\left(C_{\mathrm{e}}^{*}(X), j\right)$ (see e.g. [12, Section $4.3])$ which is a unital $C^{*}$-algebra together with a complete isometry $j$ : $X \rightarrow C_{\mathrm{e}}^{*}(X)$ with $j(1)=1$ such that $j(X)$ generates $C_{\mathrm{e}}^{*}(X)$ as a $C^{*}$-algebra, 
and possessing a certain universal property spelled out in the last reference. This $C^{*}$-algebra obviously has a canonical cone $C_{\mathrm{e}}^{*}(X)_{+}$.

COROLlary 5.7. If $X$ is a unital operator space and if $X$ also has an operator space cone $\mathfrak{c}$ which densely spans $X$, and which contains 1 , then the $C^{*}$-envelope $C_{\mathrm{e}}^{*}(X)$ (in the sense of $[12$, Section 4.3$]$ ) is also the ordered ternary envelope of $X$, with $C_{\mathrm{e}}^{*}(X)_{+}=\mathcal{T}^{\mathrm{o}}(X)_{+}$.

Proof. It is known that the unital $C^{*}$-algebra $A=C_{\mathrm{e}}^{*}(X)$ is a ternary envelope of $X$, and hence there does exist a natural cone $\mathfrak{d}$ in $A$ containing $j(\mathfrak{c})$, and therefore also containing 1 . The span $J$ of $\mathfrak{d}$ is a subTRO of $A$ containing $j(\mathfrak{c})$, and therefore containing $j(X)$, and 1 . This forces $J=A$. If $u$ is the open tripotent corresponding to $\mathfrak{d}$ in $A^{\prime \prime}$, then $A^{\prime \prime}(u)=A^{\prime \prime}$, and so $u$ is unitary. Since $1 \in \mathfrak{d}$ we have $u^{*}=u^{*} 1 \geq 0$, so that $u=1$. Thus $\mathfrak{d}=A_{+}$.

REMARK. The spaces $X$ as in the last corollary, which are also maximally ordered (resp. complete in the sense defined just above Theorem 5.3), are exactly the unital operator systems.

EXAMPLE. As a sample illustration of how our results may be applied in concrete situations, we show that if $A^{\prime}$ is the dual of a $C^{*}$-algebra $A$, with its usual cone, then there may exist no isometric positive map from $A^{\prime}$ into another $C^{*}$-algebra. (A later more elementary proof of this fact was found in [11].) We prove it in the case that $A=\ell_{2}^{\infty}$. In this case the map $j:(\alpha, \beta) \mapsto \alpha 1+\beta z$, where $z\left(e^{i \theta}\right)=e^{i \theta}$, is a unital complete isometry from $\ell_{2}^{1}$ into the $C^{*}$-algebra $B$ of continuous functions on the unit circle, and in fact the circle is well known to be the Shilov boundary of $\ell_{2}^{1}$ (see $[12$, Example 4.1.9(1)]), and so $(B, j)$ is the $C^{*}$-envelope $C_{\mathrm{e}}^{*}\left(A^{\prime}\right)$. If there existed an isometric positive map from $A^{\prime}$ into another $C^{*}$-algebra, then this map would be completely isometric, since it was noticed by Paulsen that $\ell_{2}^{1}$ has exactly one operator space structure (see [37, Proposition 3.2] for a simple proof of the latter fact). Equivalently, the usual cone on $A^{\prime}$ is an operator space cone. By the nonmatricial cone case of Corollary $5.7,(B, j)$ is also the ordered ternary envelope of $A^{\prime}$. Hence $j((0,1))=z$ is a positive function on the unit circle, which is absurd.

We remark in passing that the fact proved in the last paragraph shows that the main results about unitizations of ordered spaces in the paper [30] are not correct as stated, and this led to the correction [31].

We now turn to some other interesting conditions that a cone might satisfy, some of which also ensure that the cone is "complete" (that is, the embedding $j: X \rightarrow \mathcal{T}^{\mathrm{o}}(X)$ is a complete order embedding).

Any natural cone $\mathfrak{c}$ in a TRO $Z$ has the property that any element in $\overline{\operatorname{Span}}(\mathfrak{c})$ may be written as $x=c_{1}-c_{2}+i\left(c_{3}-c_{4}\right)$, where $c_{i} \in \mathfrak{c}$ and $c_{1}^{*} c_{2}=0$ and $c_{3}^{*} c_{4}=0$. (We remark that if $u$ is the tripotent associated with the cone, 
then the product of $c_{1}$ and $c_{2}$ in $Z(u)$ is $c_{1} u^{*} c_{2}=u u^{*} c_{1} u^{*} c_{2}=u\left(c_{1}^{\sharp}\right)^{*} c_{2}=$ $u c_{1}^{*} c_{2}$, which is 0 iff $c_{1}^{*} c_{2}=0$.) With this in mind, it is natural to consider operator space cones $\mathfrak{c}$ in an operator space $X$ with the property that any element in $\overline{\operatorname{Span}}(\mathfrak{c})$ may be written as $x=c_{1}-c_{2}+i\left(c_{3}-c_{4}\right)$, where $c_{i} \in \mathfrak{c}$ and $j\left(c_{1}\right)^{*} j\left(c_{2}\right)=0$ and $j\left(c_{3}\right)^{*} j\left(c_{4}\right)=0$. Here $j: X \rightarrow \mathcal{T}(X)$ is the Shilov boundary embedding. We say that an operator space cone is orthogonalizing if it has this property.

Proposition 5.8. Let $X$ be an operator space with an orthogonalizing operator space cone $\mathfrak{c}$. If $\mathfrak{d}$ is the natural cone of $\mathcal{T}^{\circ}(X)$, then $\mathfrak{d} \cap \overline{\operatorname{Span}}(\mathfrak{c})=\mathfrak{c}$. If in addition $\mathfrak{c}$ densely spans $X$, then $X$ is completely order embedded in $\mathcal{T}^{\circ}(X)$, and so $X$ is maximally ordered.

Proof. To see this, we view $Z=\mathcal{T}^{\circ}(X)$ as a subTRO in a $C^{*}$-algebra $B$ and $J(Z)$ as a $C^{*}$-subalgebra of $B$. We are also viewing $\overline{\operatorname{Span}}(\mathfrak{c}) \subset J(Z)$, so that, if $x \in \mathfrak{d} \cap \overline{\operatorname{Span}}(\mathfrak{c})$, we may write as above $x=c_{1}-c_{2}+i\left(c_{3}-c_{4}\right)$, with $c_{i} \in J(Z)_{+}$. Since $x=x^{*}$ in $J(Z)$, we must have $x=c_{1}-c_{2}$. Since (using the product of $J(Z)$ )

$$
0 \leq c_{2} x c_{2}=-c_{2}^{3} \leq 0
$$

we deduce that $c_{2}=0$ and $x=c_{1} \in \mathfrak{c}$. The last assertion is obvious (using also Corollary 5.6).

In a similar spirit, we remark that since the span of a natural cone in a TRO is an inner ideal, it seems of interest to consider operator space cones on an operator space $X$ such the the span of the cone is the analogue of an inner ideal in $X$. More specifically, we say that an operator space cone on $X$ is inner if $J=\overline{\operatorname{Span}}(\mathfrak{c})$ is a generalized quasi- $M$-ideal of $X$. The term "quasi$M$-ideal" is due to Kaneda [29] (and is a variant of the one-sided $M$-ideals considered e.g. in [14]). By a generalized quasi- $M$-ideal, we mean a subspace $J$ of $X$ such that the weak* closure $J^{\perp \perp}$ of $X^{\prime \prime}$, viewed as a subspace of the ternary envelope $W=\left(\mathcal{T}\left(X^{\prime \prime}\right), j\right)$ of $X^{\prime \prime}$, equals $p j\left(X^{\prime \prime}\right) q$, where $p$ and $q$ are projections on $W$ which are, respectively, right and left module maps on $W$. (In the language of [14] for example, $p$ and $q$ are orthogonal projections in $\mathcal{A}_{\ell}(W)$ and $\mathcal{A}_{r}(W)$ respectively.) The generalized quasi- $M$-ideals in a TRO are exactly the inner ideals (by e.g. the proof in the discussion before Proposition 5.2 in $[10])$.

THEOREM 5.9. Let $X$ be an operator space with an operator space cone $\mathfrak{c}$ which is inner.

(1) If $\mathfrak{d}$ is the natural cone of $\mathcal{T}^{\circ}(X)$, then $\operatorname{Span}(\mathfrak{d}) \cap X=\overline{\operatorname{Span}}(\mathfrak{c})$, and $\mathfrak{d} \cap X=\mathfrak{d} \cap \overline{\operatorname{Span}}(\mathfrak{c})$.

(2) If also $\mathfrak{c}$ is orthogonalizing then $\mathfrak{c}$ is complete (that is, the embedding of $X$ in its ordered ternary envelope $\mathcal{T}^{\mathrm{o}}(X)$ is a complete order embedding). 
Proof. Write $i_{X}: X \rightarrow X^{\prime \prime}$ for the canonical injection. We use the notation above, so that $W=\left(\mathcal{T}\left(X^{\prime \prime}\right), j\right)$ is the ternary envelope of $X^{\prime \prime}$. By [10, Lemma 5.3], $\left(\left\langle j\left(i_{X}(X)\right)\right\rangle, j \circ i_{X}\right)$ is a ternary envelope of $X$. Here $\left\langle j\left(i_{X}(X)\right)\right\rangle$ is the subTRO of $W$ generated by $j\left(i_{X}(X)\right)$. Let $E=W^{\prime \prime}$, and $Z=\left\langle j\left(i_{X}(X)\right)\right\rangle$. Then $Z^{\prime \prime} \cong Z^{\perp \perp}$ is a subWTRO of $E$. Since $Z$ is the ternary envelope of $X$, there is a natural cone $\mathfrak{d}$ on $Z$ making $Z$ the ordered ternary envelope. Let $u$ be the associated tripotent in $Z^{\prime \prime} \subset E$. For any $x \in \mathfrak{c}$ we have $p j\left(i_{X}(x)\right) q=j\left(i_{X}(x)\right)$. It follows that $\operatorname{pr}(x) q=r(x)$, and so $p u q=u$. It follows that $p z q=z$ for any $z \in Z(u)$. Thus

$$
Z(u) \cap j\left(i_{X}(X)\right) \subset p j\left(X^{\prime \prime}\right) q \cap j\left(i_{X}(X)\right)=j\left(i_{X}(J)\right) .
$$

That is, $Z(u) \cap X=J$, and $\mathfrak{d} \cap X=\mathfrak{d} \cap J$.

The second part follows from the first part and Proposition 5.8.

REMARK. It seems possible that a converse may hold, that is, if the cone is complete then it is inner.

Closing remark. In the sequel paper [11], we study the case of operator spaces $X$ which have an involution $*$ and matrix cones $\mathfrak{c}_{n} \subset M_{n}(X)_{\text {sa }}$. The morphisms in this category are all $*$-linear, of course. In this case, the ordered ternary envelope becomes a $*$-TRO, and one must use the ordered $*$-TRO theory developed in [13] in place of the ordered TRO theory in the present paper. Thus all tripotents $u$ occurring are also selfadjoint, and central in the sense that $u z=z u$ for all $z \in Z$. If $X$ is such an ordered operator space, and if $\mathcal{T}(X)$ is its ternary $*$-envelope, then there exists a natural (in the sense of [13]) cone on $\mathcal{T}(X)$ containing the (image of the) cone of $X$, and one can then take the intersection of all such natural cones to obtain the ordered ternary $*$-envelope $\mathcal{T}^{\circ}(X)$. The statement of the universal property of this envelope is similar to that of Theorem 5.3, but curiously, the proof seems to be completely different, for the reason that range tripotents need not be central. Most of our other results from this section have obvious analogues in this "selfadjoint case", which we shall not take the time to spell out. The first author began to investigate such results in discussions with Werner at the time we were working on [13].

Acknowledgements. We thank Upasana Kashyap for comments on a draft of our paper. She has also pointed out to us the validity of the analogue of [13, Theorem 4.20] for TROs and natural cones in the sense of the present paper.

\section{References}

[1] C. A. Akemann, The general Stone-Weierstrass problem, J. Funct. Anal. 4 (1969), $277-294$. 
[2] C. A. Akemann, Left ideal structure of $C^{*}$-algebras, ibid. 6 (1970), 305-317.

[3] -, A Gelfand representation theory for $C^{*}$-algebras, Pacific J. Math. 39 (1971), $1-11$.

[4] C. A. Akemann, J. Anderson, and G. K. Pedersen, Approaching infinity in $C^{*}$ algebras, J. Operator Theory 21 (1989), 255-271.

[5] C. A. Akemann and G. K. Pedersen, Facial structure in operator algebra theory, Proc. London Math. Soc. 64 (1992), 418-448.

[6] W. B. Arveson, Subalgebras of $C^{*}$-algebras, Acta Math. 123 (1969), 141-224.

[7] T. Barton and R. Timoney, Weak*-continuity of Jordan triple products and its applications, Math. Scand. 59 (1986), 177-191.

[8] M. Battaglia, Order theoretic type decomposition of $J B W^{*}$-triples, Quart. J. Math. Oxford 42 (1991), 129-147.

[9] D. P. Blecher et al., Peak tripotents (tentative title), work in progress.

[10] D. P. Blecher, D. M. Hay, and M. Neal, Hereditary subalgebras of operator algebras, J. Operator Theory, to appear.

[11] D. P. Blecher, K. Kirkpatrick, M. Neal, and W. Werner, Ordered involutive operator spaces, Positivity 11 (2007), 497-510.

[12] D. P. Blecher and C. Le Merdy, Operator Algebras and their Modules - an Operator Space Approach, Oxford Univ. Press, Oxford, 2004.

[13] D. P. Blecher and W. Werner, Ordered $C^{*}$-modules, Proc. London Math. Soc. 92 (2006), 682-712.

[14] D. P. Blecher and V. Zarikian, The calculus of one-sided $M$-ideals and multipliers in operator spaces, Mem. Amer. Math. Soc. 842 (2006).

[15] L. J. Bunce, F. J. Fernández-Polo, J. Martinez-Moreno, and A. M. Peralta, A SaitoTomita-Lusin theorem for JB*-triples and applications, Quart. J. Math. 57 (2006), $37-48$.

[16] M.-D. Choi and E. G. Effros, Injectivity and operator spaces, J. Funct. Anal. 24 (1977), 156-209.

[17] C. M. Edwards and G. T. Rüttimann, On the facial structure of the unit balls in a $J B W^{*}$-triple and its predual, J. London Math. Soc. 38 (1988), 317-332.

[18] -, -, Inner ideals in $W^{*}$-algebras, Michigan Math. J. 36 (1989), 147-159.

[19] -, - Inner ideals in $C^{*}$-algebras, Math. Ann. 290 (1991), 621-628.

[20] —, -, On inner ideals in ternary algebras, Math. Z. 204 (1990), 309-318.

[21] —, Compact tripotents in bi-dual JB*-triples, Math. Proc. Cambridge Philos. Soc. 120 (1996), 155-173.

[22] E. G. Effros, Order ideals in a $C^{*}$-algebra and its dual, Duke Math. J. 30 (1963), 391-411.

[23] F. J. Fernández-Polo and A. M. Peralta, Compact tripotents and the Stone-Weierstrass theorem for $C^{*}$-algebras and $J B^{*}$-triples, J. Operator Theory 58 (2007), 157 173.

[24] - - - Closed tripotents and weak compactness in the dual space of a JB*-triple, J. London Math. Soc. 74 (2006), 75-92.

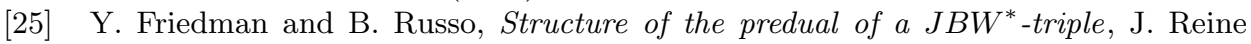
Angew. Math. 356 (1985), 67-89.

[26] M. Hamana, Triple envelopes and Silov boundaries of operator spaces, Math. J. Toyama Univ. 22 (1999), 77-93.

[27] D. M. Hay, Closed projections and peak interpolation for operator algebras, Integral Equations Operator Theory 57 (2007), 491-512.

[28] G. Horn, Characterization of the predual and ideal structure of a $J B W^{*}$-triple, Math. Scand. 61 (1987), 117-133. 
[29] M. Kaneda, unpublished note on quasi- $M$-ideals, 2003.

[30] A. K. Karn, Adjoining an order unit to a matrix ordered space, Positivity 9 (2005), $207-223$.

[31] A. K. Karn, Corrigendum to the paper "Adjoining an order unit to a matrix ordered space", ibid. 11 (2007), 367-374.

[32] K. Kirkpatrick, The Shilov boundary and $M$-structure of operator spaces, Research Experiences for Undergraduates paper, Houston, 2001.

[33] M. Neal, Inner ideals and facial structure of the quasi-state space of a JB-algebra, J. Funct. Anal. 173 (2000), 284-307.

[34] M. Neal and B. Russo, Operator space characterizations of $C^{*}$-algebras and ternary rings, Pacific J. Math. 209 (2003), 339-364.

[35] - - - State spaces of JB*-triples, Math. Ann. 328 (2004), 585-624.

[36] G. K. Pedersen, $C^{*}$-algebras and their Automorphism Groups, Academic Press, London, 1979.

[37] G. Pisier, Introduction to Operator Space Theory, London Math. Soc. Lecture Note Ser. 294, Cambridge Univ. Press, Cambridge, 2003.

[38] B. Russo, Structure of JB*-triples, in: Jordan Algebras (Oberwolfach, 1992), W. Kaup et al. (eds.), de Gruyter, 1994, 209-280.

[39] W. Werner, Subspaces of $L(H)$ that are *-invariant, J. Funct. Anal. 193 (2002), $207-223$.

[40] -, Multipliers on matrix ordered operator spaces and some K-groups, ibid. 206 (2004), 356-378.

Department of Mathematics

University of Houston

Houston, TX 77204-3008, U.S.A.

E-mail: dblecher@math.uh.edu
Department of Mathematics

Denison University

Granville, OH 43023, U.S.A.

E-mail: nealm@denison.edu

Received November 28, 2006

Revised version July 17, 2007 